\title{
Shockingly low water abundances in Herschel/PACS observations of low-mass protostars in Perseus ${ }^{\star}$
}

\author{
A. Karska ${ }^{1,2,3}$, L. E. Kristensen ${ }^{4}$, E. F. van Dishoeck ${ }^{1,2}$, M. N. Drozdovskaya ${ }^{2}$, J. C. Mottram ${ }^{2}$, G. J. Herczeg ${ }^{5}$,
} S. Bruderer ${ }^{1}$, S. Cabrit ${ }^{6}$, N. J. Evans II ${ }^{7}$, D. Fedele ${ }^{1}$, A. Gusdorf ${ }^{8}$, J. K. Jørgensen ${ }^{9,10}$, M. J. Kaufman ${ }^{11}$, G. J. Melnick ${ }^{3}$, D. A. Neufeld ${ }^{12}$, B. Nisini ${ }^{13}$, G. Santangelo ${ }^{13}$, M. Tafalla ${ }^{14}$, and S. F. Wampfler ${ }^{9}, 10$

\footnotetext{
${ }^{1}$ Max-Planck Institut für Extraterrestrische Physik (MPE), Giessenbachstr. 1, 85748 Garching, Germany e-mail: agata.karska@gmail.com

2 Leiden Observatory, Leiden University, PO Box 9513, 2300 RA Leiden, The Netherlands

3 Astronomical Observatory, Adam Mickiewicz University, Słoneczna 36, 60-268 Poznań, Poland

${ }^{4}$ Harvard-Smithsonian Center for Astrophysics, 60 Garden Street, Cambridge, MA 02138, USA

5 Kavli Institut for Astronomy and Astrophysics, Yi He Yuan Lu 5, HaiDian Qu, Peking University, 100871 Beijing, PR China

${ }^{6}$ LERMA, UMR 8112 du CNRS, Observatoire de Paris, École Normale Supérieure, Université Pierre et Marie Curie, Université de Cergy-Pontoise, 61 Av. de l'Observatoire, 75014 Paris, France

7 Department of Astronomy, The University of Texas at Austin, 2515 Speedway, Stop C1400, Austin, TX 78712-1205, USA

8 LERMA, UMR 8112 du CNRS, Observatoire de Paris, École Normale Supérieure, 24 rue Lhomond, 75231 Paris Cedex 05, France

Niels Bohr Institute, University of Copenhagen, Juliane Maries Vej 30, 2100 Copenhagen Ø., Denmark

10 Centre for Star and Planet Formation, Natural History Museum of Denmark, University of Copenhagen, Øster Voldgade 5-7, 1350 Copenhagen K., Denmark

11 Department of Physics, San Jose State University, One Washington Square, San Jose, CA 95192-0106, USA

12 Department of Physics and Astronomy, Johns Hopkins University, 3400 North Charles Street, Baltimore, MD 21218, USA

13 INAF - Osservatorio Astronomico di Roma, 00040 Monte Porzio Catone, Italy

14 Observatorio Astronómico Nacional (IGN), Calle Alfonso XII,3. 28014 Madrid, Spain
}

Received 9 May 2014 / Accepted 15 September 2014

\section{ABSTRACT}

\begin{abstract}
Context. Protostars interact with their surroundings through jets and winds impinging on the envelope and creating shocks, but the nature of these shocks is still poorly understood.

Aims. Our aim is to survey far-infrared molecular line emission from a uniform and significant sample of deeply-embedded low-mass young stellar objects (YSOs) in order to characterize shocks and the possible role of ultraviolet radiation in the immediate protostellar environment.

Methods. Herschel/PACS spectral maps of 22 objects in the Perseus molecular cloud were obtained as part of the William Herschel Line Legacy (WILL) survey. Line emission from $\mathrm{H}_{2} \mathrm{O}, \mathrm{CO}$, and $\mathrm{OH}$ is tested against shock models from the literature.

Results. Observed line ratios are remarkably similar and do not show variations with physical parameters of the sources (luminosity, envelope mass). Most ratios are also comparable to those found at off-source outflow positions. Observations show good agreement with the shock models when line ratios of the same species are compared. Ratios of various $\mathrm{H}_{2} \mathrm{O}$ lines provide a particularly good diagnostic of pre-shock gas densities, $n_{\mathrm{H}} \sim 10^{5} \mathrm{~cm}^{-3}$, in agreement with typical densities obtained from observations of the postshock gas when a compression factor on the order of 10 is applied (for non-dissociative shocks). The corresponding shock velocities, obtained from comparison with $\mathrm{CO}$ line ratios, are above $20 \mathrm{~km} \mathrm{~s}^{-1}$. However, the observations consistently show $\mathrm{H}_{2} \mathrm{O}-$ to-CO and $\mathrm{H}_{2} \mathrm{O}$-to-OH line ratios that are one to two orders of magnitude lower than predicted by the existing shock models.

Conclusions. The overestimated model $\mathrm{H}_{2} \mathrm{O}$ fluxes are most likely caused by an overabundance of $\mathrm{H}_{2} \mathrm{O}$ in the models since the excitation is well-reproduced. Illumination of the shocked material by ultraviolet photons produced either in the star-disk system or, more locally, in the shock, would decrease the $\mathrm{H}_{2} \mathrm{O}$ abundances and reconcile the models with observations. Detections of hot $\mathrm{H}_{2} \mathrm{O}$ and strong $\mathrm{OH}$ lines support this scenario.
\end{abstract}

Key words. astrochemistry - stars: formation - ISM: jets and outflows

\section{Introduction}

Shocks are ubiquitous phenomena where outflow-envelope interactions take place in young stellar objects (YSO). Large-scale shocks are caused by the bipolar jets and protostellar winds impinging on the envelope along the cavity walls carved by the

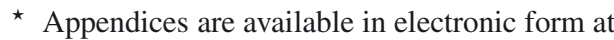
http://www . aanda.org passage of the jet (Arce et al. 2007; Frank et al. 2014). This important interaction needs to be characterized in order to understand and quantify the feedback from protostars onto their surroundings and, ultimately, to explain the origin of the initial mass function, disk fragmentation, and the binary fraction.

Theoretically, shocks are divided into two main types based on a combination of magnetic field strength, shock velocity, density, and level of ionization (Draine 1980; Draine et al. 1983; Hollenbach et al. 1989; Hollenbach 1997). In continuous 
( $C$-type) shocks, in the presence of a magnetic field and low ionization, the weak coupling between the ions and neutrals results in a continuous change in the gas parameters. Peak temperatures of a few $10^{3} \mathrm{~K}$ allow the molecules to survive the passage of the shock, which is therefore referred to as non-dissociative. In jump ( $J$-type) shocks, physical conditions change in a discontinuous way, leading to higher peak temperatures than in $C$ shocks of the same speed and for a given density. Depending on the shock velocity, $J$ shocks are either non-dissociative (velocities below about $\sim 30 \mathrm{~km} \mathrm{~s}^{-1}$, peak temperatures of a few $10^{4} \mathrm{~K}$ ) or dissociative (peak temperatures even exceeding $10^{5} \mathrm{~K}$ ), but the molecules efficiently reform in the post-shock gas.

Shocks reveal their presence most prominently in the infrared (IR) domain, where the post-shock gas is efficiently cooled by numerous atomic and molecular emission lines. Cooling from $\mathrm{H}_{2}$ is dominant in outflow shocks (Nisini et al. 2010b; Giannini et al. 2011b), but its mid-IR emission is strongly affected by extinction in the dense envelopes of young protostars (Giannini et al. 2001a; Nisini et al. 2002; Davis et al. 2008; Maret et al. 2009). In the far-IR, rotational transitions of water vapor $\left(\mathrm{H}_{2} \mathrm{O}\right)$ and carbon monoxide $(\mathrm{CO})$ are predicted to play an important role in the cooling process (Goldsmith \& Langer 1978; Neufeld \& Dalgarno 1989; Hollenbach 1997) and can serve as a diagnostic of the shock type, its velocity, and the preshock density of the medium (Hollenbach et al. 1989; Kaufman \& Neufeld 1996; Flower \& Pineau des Forêts 2010, 2012).

The first observations of the critical wavelength regime to test these models $(\lambda \sim 45-200 \mu \mathrm{m})$ were taken using the LongWavelength Spectrometer (LWS, Clegg et al. 1996) on board the Infrared Space Observatory (ISO, Kessler et al. 1996). Far-IR atomic and molecular emission lines were detected toward several low-mass deeply-embedded protostars (Nisini et al. 2000; Giannini et al. 2001a; van Dishoeck 2004), but its origin was unclear because of the poor spatial resolution of the telescope ( 80", Nisini et al. 2002; Ceccarelli et al. 2002).

The sensitivity and spectral resolution of the Photodetector Array Camera and Spectrometer (PACS, Poglitsch et al. 2010) on board Herschel allowed a significant increase in the number of detections of far-IR lines in young protostars compared with the early ISO results and has revealed rich molecular and atomic line emission both at the protostellar (e.g., van Kempen et al. 2010; Goicoechea et al. 2012; Herczeg et al. 2012; Visser et al. 2012; Green et al. 2013; Karska et al. 2013; Lindberg et al. 2014; Manoj et al. 2013; Wampfler et al. 2013) and at pure outflow positions (Santangelo et al. 2012, 2013; Codella et al. 2012; Lefloch et al. 2012a; Vasta et al. 2012; Nisini et al. 2013).

The unprecedented spatial resolution of PACS allowed detailed imaging of L1157 providing firm evidence that most far-IR $\mathrm{H}_{2} \mathrm{O}$ emission originates in the outflows (Nisini et al. 2010a). A mapping survey of about 20 protostars revealed similarities between the spatial extent of $\mathrm{H}_{2} \mathrm{O}$ and high- $J \mathrm{CO}$ (Karska et al. 2013). Additional strong flux correlations between these species and similarities in the velocity-resolved profiles (Kristensen et al. 2010, 2012; San José-García et al. 2013; Santangelo et al. 2014) suggest that the emission from the two molecules arises from the same regions. This is further confirmed by finely-spatially sampled PACS maps in CO 16-15 and various $\mathrm{H}_{2} \mathrm{O}$ lines in shock positions of L1448 and L1157 (Santangelo et al. 2013; Tafalla et al. 2013). On the other hand, the spatial extent of $\mathrm{OH}$ resembles the extent of $[\mathrm{OI}]$ and, additionally, a strong flux correlation between the two species is found (Karska et al. 2013; Wampfler et al. 2013). Therefore, at least part of the $\mathrm{OH}$ emission most likely originates in a dissociative $J$-shock, together with [O I] (Wampfler et al. 2010, 2013; Benedettini et al. 2012).

To date, comparisons of the far-IR observations with shock models have been limited to a single source or its outflow positions (e.g., Nisini et al. 1999; Benedettini et al. 2012; Vasta et al. 2012; Santangelo et al. 2012; Dionatos et al. 2013; Lee et al. 2013; Flower \& Pineau des Forêts 2013). Even in these studies, separate analysis of each species (or different pairs of species) often led to different sets of shock properties that needed to be reconciled. For example, Dionatos et al. (2013) show that $\mathrm{CO}$ and $\mathrm{H}_{2}$ line emission in Serpens SMM3 originates from a $20 \mathrm{~km} \mathrm{~s}^{-1} J$ shock at low pre-shock densities $\left(\sim 10^{4} \mathrm{~cm}^{-3}\right)$, but the $\mathrm{H}_{2} \mathrm{O}$ and $\mathrm{OH}$ emission is better explained by a $30-40 \mathrm{~km} \mathrm{~s}^{-1}$ $C$ shock. In contrast, Lee et al. (2013) associate emission from both $\mathrm{CO}$ and $\mathrm{H}_{2} \mathrm{O}$ in L1448-MM with a $40 \mathrm{~km} \mathrm{~s}^{-1} C$ shock at high pre-shock densities $\left(\sim 10^{5} \mathrm{~cm}^{-3}\right)$, consistent with the analysis of the L1448-R4 outflow position (Santangelo et al. 2013). Analysis restricted to $\mathrm{H}_{2} \mathrm{O}$ lines alone often indicates an origin in non-dissociative $J$ shocks (Santangelo et al. 2012; Vasta et al. 2012; Busquet et al. 2014), while separate analysis of $\mathrm{CO}, \mathrm{OH}$, and atomic species favors dissociative $J$ shocks (Benedettini et al. 2012; Lefloch et al. 2012b). The question remains how to break degeneracies between these models and how typical the derived shock properties are for young protostars. In addition, surveys of high- $J$ CO lines with PACS have revealed two universal temperature components in the CO ladder toward all deeplyembedded low-mass protostars (Herczeg et al. 2012; Goicoechea et al. 2012; Green et al. 2013; Karska et al. 2013; Kristensen et al. 2013; Manoj et al. 2013; Lee et al. 2013). The question is how the different shock properties relate with the "warm" $(T \sim 300 \mathrm{~K})$ and "hot" $(T \gtrsim 700 \mathrm{~K})$ components seen in the $\mathrm{CO}$ rotational diagrams.

In this paper, far-IR spectra of 22 low-mass YSOs observed as part of the William Herschel Line Legacy (WILL) survey (PI: E. F. van Dishoeck) are compared to the shock models from Kaufman \& Neufeld (1996) and Flower \& Pineau des Forêts (2010). All sources are confirmed deeply-embedded YSOs located in the well-studied Perseus molecular cloud spanning the Class 0 and I regime (Knee \& Sandell 2000; Enoch et al. 2006, 2009; Jørgensen et al. 2006, 2007; Hatchell et al. 2007a,b; Davis et al. 2008; Arce et al. 2010). $\mathrm{H}_{2} \mathrm{O}, \mathrm{CO}$, and $\mathrm{OH}$ lines are analyzed together for this uniform sample to answer the following questions: Do far-IR line observations agree with the shock models? How much variation in observational diagnostics of shock conditions is found between different sources? Can one set of shock parameters explain all molecular species and transitions? Are there systematic differences between shock characteristics inferred using the CO lines from the "warm" and "hot" components? How do shock conditions vary with the distance from the powering protostar?

This paper is organized as follows. Section 2 describes our source sample, instrument with adopted observing mode, and reduction methods. Section 3 presents the results of the observations: line and continuum maps, and the extracted spectra. Section 4 shows comparison between the observations and shock models. Section 5 discusses results obtained in Sects. 4 and 6 presents the conclusions.

\section{Observations}

All observations presented here were obtained as part of the "William Herschel Line Legacy" (WILL) OT2 program on Herschel (Mottram et al., in prep.). The WILL survey is a study of $\mathrm{H}_{2} \mathrm{O}$ lines and related species with PACS and the Heterodyne 
Table 1. Catalog information and source properties.

\begin{tabular}{|c|c|c|c|c|c|c|c|}
\hline Object & 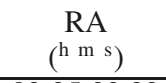 & $\begin{array}{c}\text { Dec } \\
\left({ }^{\circ}, \prime \prime\right)\end{array}$ & $\begin{array}{r}T_{\text {bol }} \\
(\mathrm{K}) \\
\end{array}$ & $\begin{array}{r}L_{\text {bol }} \\
\left(L_{\odot}\right) \\
\end{array}$ & $\begin{array}{l}M_{\text {env }} \\
\left(M_{\odot}\right) \\
\end{array}$ & Region & Other names \\
\hline Per01 & $03: 25: 22.32$ & $+30: 45: 13.9$ & 44 & 4.5 & 1.14 & L1448 & Per-emb 22, L1448 IRS2, IRAS03222+3034, YSO 1 \\
\hline Per02 & $03: 25: 36.49$ & $+30: 45: 22.2$ & 50 & 10.6 & 3.17 & L1448 & Per-emb 33, L1448 N(A), L1448 IRS3, YSO 2 \\
\hline Per03 ${ }^{a}$ & $03: 25: 39.12$ & $+30: 43: 58.2$ & 47 & 8.4 & 2.56 & L1448 & Per-emb 42, L1448 MMS, L1448 C(N), YSO 3 \\
\hline Per04 & $03: 26: 37.47$ & $+30: 15: 28.1$ & 61 & 1.2 & 0.29 & L1451 & Per-emb 25, IRAS03235+3004, YSO 4 \\
\hline Per05 & 03:28:37.09 & $+31: 13: 30.8$ & 85 & 11.1 & 0.35 & NGC 1333 & Per-emb 35, NGC 1333 IRAS1, IRAS03255+3103, YSO 11 \\
\hline Per06 & $03: 28: 57.36$ & $+31: 14: 15.9$ & 85 & 6.9 & 0.30 & NGC 1333 & Per-emb 36, NGC 1333 IRAS2B, YSO 16 \\
\hline Per07 & 03:29:00.55 & $+31: 12: 00.8$ & 37 & 0.7 & 0.32 & NGC 1333 & Per-emb 3, HRF 65, YSO 18 \\
\hline Per08 & 03:29:01.56 & $+31: 20: 20.6$ & 131 & 16.8 & 0.86 & NGC 1333 & Per-emb 54, HH 12, YSO 19 \\
\hline Per09 & 03:29:07.78 & $+31: 21: 57.3$ & 128 & 23.2 & 0.24 & NGC 1333 & Per-emb 50 \\
\hline Per10 & 03:29:10.68 & $+31: 18: 20.6$ & 45 & 6.9 & 1.37 & NGC 1333 & Per-emb 21, HRF 46, YSO 23 \\
\hline Per11 ${ }^{b}$ & 03:29:12.06 & $+31: 13: 01.7$ & 28 & 4.4 & 5.42 & NGC 1333 & Per-emb 13, NGC 1333 IRAS4B', YSO 25 \\
\hline Per12 & $03: 29: 13.54$ & $+31: 13: 58.2$ & 31 & 1.1 & 1.30 & NGC 1333 & Per-emb 14, NGC 1333 IRAS4C, YSO 26 \\
\hline Per13 & 03:29:51.82 & $+31: 39: 06.0$ & 40 & 0.7 & 0.51 & NGC 1333 & Per-emb 9, IRAS03267+3128, YSO 31 \\
\hline Per14 & 03:30:15.14 & $+30: 23: 49.4$ & 88 & 1.8 & 0.14 & B1-ridge & Per-emb 34, IRAS03271+3013 \\
\hline Per15 & 03:31:20.98 & $+30: 45: 30.1$ & 35 & 1.7 & 1.29 & B1-ridge & Per-emb 5, IRAS03282+3035, YSO 32 \\
\hline Per16 & 03:32:17.96 & $+30: 49: 47.5$ & 30 & 1.1 & 2.75 & B1-ridge & Per-emb 2, IRAS03292+3039, YSO 33 \\
\hline Per17 ${ }^{c}$ & 03:33:14.38 & $+31: 07: 10.9$ & 43 & 0.7 & 1.20 & B1 & Per-emb 6, B1 SMM3, YSO 35 \\
\hline Per1 $18^{c}$ & 03:33:16.44 & $+31: 06: 52.5$ & 25 & 1.1 & 1.22 & B1 & Per-emb 10, B1 d, YSO 36 \\
\hline Per19 & 03:33:27.29 & $+31: 07: 10.2$ & 93 & 1.1 & 0.23 & B1 & Per-emb 30, B1 SMM11, YSO 40 \\
\hline Per20 & $03: 43: 56.52$ & $+32: 00: 52.8$ & 27 & 2.3 & 2.05 & IC 348 & Per-emb 1, HH 211 MMS, YSO 44 \\
\hline Per21 & 03:43:56.84 & $+32: 03: 04.7$ & 34 & 2.1 & 1.88 & IC 348 & Per-emb 11, IC348 MMS, IC348 SW, YSO 43 \\
\hline Per22 & 03:44:43.96 & $+32: 01: 36.2$ & 43 & 2.6 & 0.64 & IC 348 & Per-emb 8, IC348 a, IRAS03415+3152, YSO 48 \\
\hline
\end{tabular}

Notes. Bolometric temperatures and luminosities are determined including the PACS continuum values; the procedure will be discussed in Mottram et al. (in prep.). Numbered Per-emb names come from Enoch et al. (2009), whereas the numbered YSO names come from Jørgensen et al. (2006) and were subsequently used in Davis et al. (2008). Other source identifiers were compiled using Jørgensen et al. (2007), Rebull et al. (2007), Davis et al. (2008), and Velusamy et al. (2014). Observations obtained in 2011 which overlap with Per03 and Per11 are presented in Lee et al. (2013) and Herczeg et al. (2012), respectively. ${ }^{(a)}$ Tabulated values come from Green et al. (2013), where full PACS spectra are obtained. The WILL values for $T_{\text {bol }}$ and $L_{\text {bol }}$ are $48 \mathrm{~K}$ and $8.0 L_{\odot}$, respectively, within $5 \%$ of those of Green et al. (2013). ${ }^{(b)}$ Tabulated values come from Karska et al. (2013) and agree within $2 \%$ of the values $29 \mathrm{~K}$ and $4.3 L_{\odot}$ obtained here. We prefer to use the previously published values because the pointing of that observation was better centered on the IRAS4B. ${ }^{(b)}$ The off-positions of Per17 and Per18 were contaminated by other continuum sources, and therefore the $T_{\text {bol }}$ and $L_{\text {bol }}$ are calculated here without the Herschel/PACS points.

Instrument for the Far-Infrared (HIFI, de Graauw et al. 2010) toward an unbiased flux-limited sample of low-mass protostars newly discovered in the recent Spitzer (c2d, Gutermuth et al. 2009, 2010; Evans et al. 2009) and Herschel (André et al. 2010) Gould Belt imaging surveys. Its main aim is to study the physics and chemistry of star-forming regions in a statistically significant way by extending the sample of low-mass protostars observed in the "Water in star-forming regions with Herschel" (WISH, van Dishoeck et al. 2011) and "Dust, Ice, and Gas in Time" (DIGIT, Green et al. 2013) programs.

This paper presents the Herschel/PACS spectra of 22 lowmass deeply-embedded YSOs located exclusively in the Perseus molecular cloud (see Table 1) to ensure the homogeneity of the sample (similar ages, environment, and distance). The sources were selected from the combined SCUBA and Spitzer/IRAC and MIPS catalog of Jørgensen et al. (2007) and Enoch et al. (2009), and all contain a confirmed embedded YSO (Stage 0 or I, Robitaille et al. 2006, 2007) in the center.

The WILL sources were observed using the line spectroscopy mode on PACS which offers deep integrations and finely sampled spectral resolution elements (minimum 3 samples per FWHM depending on the grating order, PACS Observer's Manual $\left.^{1}\right)$ over short wavelength ranges $(0.5-2 \mu \mathrm{m})$. The line selection was based on the prior experience with the PACS spectra obtained over the full far-infrared spectral range in the WISH and DIGIT programs and is summarized in Table A.1. Details of

\footnotetext{
1 http://herschel.esac.esa.int/Docs/PACS/html/pacs_om. html
}

the observations of the Perseus sources within the WILL survey are shown in Table A.2.

PACS is an integral field unit with a $5 \times 5$ array of spatial pixels (hereafter spaxels) covering a field of view of $\sim 47^{\prime \prime} \times 47^{\prime \prime}$. Each spaxel measured $\sim 9.4^{\prime \prime} \times 9.4^{\prime \prime}$, or $2 \times 10^{-9} \mathrm{sr}$, and at the distance to Perseus ( $d=235$ pc, Hirota et al. 2008) resolves emission down to 2300 AU. The total field of view is about $5.25 \times 10^{-8} \mathrm{sr}$ and $\sim 11000$ AU. The properly fluxcalibrated wavelength ranges include: $\sim 55-70 \mu \mathrm{m}, \sim 72-94 \mu \mathrm{m}$, and $\sim 105-187 \mu \mathrm{m}$, corresponding to the second $(<100 \mu \mathrm{m})$ and first spectral orders $(>100 \mu \mathrm{m})$. Their respective spectral resolving power are $R \sim 2500-4500$ (velocity resolution of $\left.\Delta v \sim 70-120 \mathrm{~km} \mathrm{~s}^{-1}\right), 1500-2500\left(\Delta v \sim 120-200 \mathrm{~km} \mathrm{~s}^{-1}\right)$, and 1000-1500 ( $\left.\Delta v \sim 200-300 \mathrm{~km} \mathrm{~s}^{-1}\right)$. The standard choppingnodding mode was used with a medium $\left(3^{\prime}\right)$ chopper throw. The telescope pointing accuracy is typically better than $2^{\prime \prime}$ and can be evaluated to first order using the continuum maps.

The basic data reduction presented here was performed using the Herschel Interactive Processing Environment v.10 (HIPE, Ott 2010). The flux was normalized to the telescopic background and calibrated using observations of Neptune. Spectral flatfielding within HIPE was used to increase the signal-to-noise (for details, see Herczeg et al. 2012; Green et al. 2013). The overall flux calibration is accurate to $\sim 20 \%$, based on the flux repeatability for multiple observations of the same target in different programs, cross-calibrations with HIFI and ISO, and continuum photometry.

Custom IDL routines were used to extract fluxes using Gaussian fits with fixed line width (for details, see Herczeg et al. 2012). The total, $5 \times 5$ line fluxes were calculated by 
Table 2. Notes on mapped regions for individual sources.

\begin{tabular}{|c|c|c|c|c|c|c|c|c|c|}
\hline \multirow[t]{2}{*}{$\overline{\text { Object }}$} & \multicolumn{2}{|c|}{ " Continuum } & \multicolumn{2}{|c|}{ Line } & \multicolumn{2}{|c|}{ Sources } & \multicolumn{2}{|c|}{ Outflows } & \multirow[t]{2}{*}{ Remarks } \\
\hline & on & off & on & off & single & multiple & single & multiple & \\
\hline Per01 & $\mathrm{X}$ & - & $\mathrm{X}$ & - & $\mathrm{X}$ & - & $\mathrm{X}$ & - & \multirow{5}{*}{$\begin{array}{l}\text { three sources }(3) \text {, contam. by Per03 }(1,2) \\
\text { a binary }(1,4)\end{array}$} \\
\hline Per02 & $X$ & - & - & $X$ & - & $X$ & - & Xc & \\
\hline Per03 & - & $\mathrm{X}$ & $\mathrm{Xe}$ & & - & $X$ & - & $X$ & \\
\hline Per04 & $X$ & - & nd & nd & $\mathrm{X}$ & - & $\mathrm{X}$ & - & \\
\hline Per05 & $\mathrm{X}$ & - & $X$ & - & $\mathrm{X}$ & - & $X$ & - & \\
\hline Per06 & - & $\mathrm{X}$ & - & $\mathrm{X}$ & - & $\mathrm{X}$ ? & - & $\mathrm{Xc}$ & \multirow{4}{*}{$\begin{array}{c}\text { contam. by NGC } 1333 \text { IRAS } 2 \mathrm{~A} \text { in N-W (5) } \\
\text { contam. in lines in N-W }\end{array}$} \\
\hline Per07 & $X$ & - & nd & nd & $\mathrm{X}$ & - & $\mathrm{Xc}$ & - & \\
\hline Per08 & - & $X$ & $\mathrm{Xe}$ & - & $X$ & - & $X$ & - & \\
\hline Per09 & $\mathrm{X}$ & - & $\mathrm{Xe}$ & - & $\mathrm{X}$ ? & - & $\mathrm{X}$ ? & - & \\
\hline Per10 & - & $X$ & - & $X$ & - & $\mathrm{X}$ & - & $\mathrm{Xc}$ & \multirow{2}{*}{$\frac{\text { two sources }(1,2), \text { dominated by YSO } 24(1,2)}{\text { NGC } 1333 \text { IRAS4A in N-W }(5)}$} \\
\hline Per11 & - & $\mathrm{X}$ & $\mathrm{Xe}$ & - & - & $\mathrm{X}$ & - & $\mathrm{Xc}$ & \\
\hline Per12 & $X$ & - & nd & nd & $X$ & - & $\mathrm{Xc}$ & - & \multirow{4}{*}{ contam. in lines by NGC 1333 IRAS4A in N-W (5) } \\
\hline Per13 & $\mathrm{X}$ & - & nd & nd & $\mathrm{X}$ ? & - & $X$ ? & & \\
\hline Per14 & $\mathrm{X}$ & - & $\mathrm{X}$ & - & $X ?$ & - & $\mathrm{X}$ ? & & \\
\hline Per15 & $X$ & - & $\mathrm{X}$ & - & $X$ & - & $X$ & - & \\
\hline Per16 & $\mathrm{X}$ & - & nd & nd & $\mathrm{X}$ & - & $\mathrm{X}$ & - & \multirow{5}{*}{$\begin{array}{l}\text { Per18 in S-E, nodded on emission } \\
\text { B1-b outflow? (2) }\end{array}$} \\
\hline Per17 & - & $X ?$ & nd & nd & - & $X$ & - & $\mathrm{Xc}$ ? & \\
\hline Per18 & $X$ & - & $\mathrm{X}$ & - & $X$ & - & - & Xc? & \\
\hline Per19 & $\mathrm{X}$ & - & $\mathrm{X}$ & - & $\mathrm{X}$ & - & $X$ & - & \\
\hline Per20 & $\mathrm{X}$ & - & $\mathrm{Xe}$ & - & $\mathrm{X}$ & - & $X$ & - & \\
\hline Per21 & $\mathrm{X}$ & - & $\mathrm{Xe}$ & - & $\mathrm{X}$ ? & - & $\mathrm{X}$ ? & - & \\
\hline Per22 & X & - & $\mathrm{Xe}$ & - & $X ?$ & - & $X$ ? & - & \\
\hline
\end{tabular}

Notes. Columns 2-5 indicate the location of the continuum at $179 \mu \mathrm{m}$ and line emission peak of the $\mathrm{H}_{2} \mathrm{O} 22_{12}-1_{01}(179.527 \mu \mathrm{m})$ transition on the maps (whether on or off-center). Columns 6-9 provide information about the number of sources and their outflows in the mapped region. Contamination by the outflows driven by sources outside the PACS field of view is mentioned in the last column. Extended emission associated with the targeted sources is denoted by "e". Non-detections are abbreviated by "nd", while "c" marks the contamination of the targeted source map by outflows from other sources.

References. (1) Jørgensen et al. (2006); (2) Davis et al. (2008); (3) Looney et al. (2000); (4) Hirano et al. (2010); Lee et al. (2013); (5) Y1ldı et al. (2014).

co-adding spaxels with detected line emission, after excluding contamination from other nearby sources except Per 2, 3, 10 , and 18, where spatial separation between different components is too small (see Sect. 3.1). For sources showing extended emission, the set of spaxels providing the maximum flux was chosen for each line separately. For point-like sources, the flux is calculated at the central position and then corrected for the PSF using wavelength-dependent correction factors (see PACS Observer's Manual). Table A.3 shows the line detections toward each source, while the actual fluxes will be tabulated in the forthcoming paper for all the WILL sources (Karska et al., in prep.). Fits cubes containing the spectra in each spaxel will be available for download in early 2015 at http: //www . strw . leidenuniv.nl/WISH/.

\section{Results}

In the following sections, PACS lines and maps of the Perseus YSOs are presented. Most sources in this sample show emission in just the central spaxel. Only a few sources show extended emission and those maps are compared to maps at other wavelengths to check for possible contamination by other sources and their outflows. In this way, the spaxels of the maps with emission originating from our objects are established and line fluxes determined over those spaxels. The emergent line spectra are then discussed.

\subsection{Spatial extent of line emission}

Table 2 provides a summary of the patterns of $\mathrm{H}_{2} \mathrm{O} 22_{12}-1_{01}$ line and continuum emission at $179 \mu \mathrm{m}$ for all WILL sources in Perseus (maps are shown in Figs. A.1 and A.2). The mid-infrared continuum and $\mathrm{H}_{2}$ line maps from Jørgensen et al. (2006) and Davis et al. (2008, including CO 3-2 observations from Hatchell et al. 2007a) are used to obtain complementary information on the sources and their outflows. For a few well-known outflow sources, large-scale CO 6-5 maps from Y1ld1z et al. (2014) are also considered.

As shown in Table 2, the majority of the PACS maps toward Perseus YSOs do not show any extended line emission. The well-centered continuum and line emission originates from a single object and an associated bipolar outflow for 12 out of 22 sources. Among the sources with spatially-resolved extended emission on the maps, various reasons are identified for their origin as illustrated in Fig. 1. In the map of Per 2, contribution from three nearby protostars and a strong outflow from the more distant L1448-MM source cause the extended line and continuum pattern. Emission in the Per 12 map is detected away from the continuum peak, but the emission originates from a large-scale outflow from NGC 1333-IRAS4A, not the targeted source. The $\mathrm{H}_{2} \mathrm{O}$ emission in the Per 20 map is detected in the direction of the strong outflow and its extent is only slightly affected by the small mispointing revealed by the asymmetric continuum emission.

Extended emission beyond the well-centered continuum, as in the case of Per 20, is seen clearly only in Per 9 and Per 21-22. Additionally, the continuum peaks for Per 3, 8, and 11 are offcenter, whereas the line emission peaks on-source, suggesting that some extended line emission is associated with the source itself and not only due to the mispointing ${ }^{2}$. Similar continuum patterns are seen in Per 6 and Per 10, but here the line emission

2 On-source observations that contained Per 3 and Per 11 in the same field of view are discussed in separate papers by Lee et al. (2013) and Herczeg et al. (2012), respectively. 

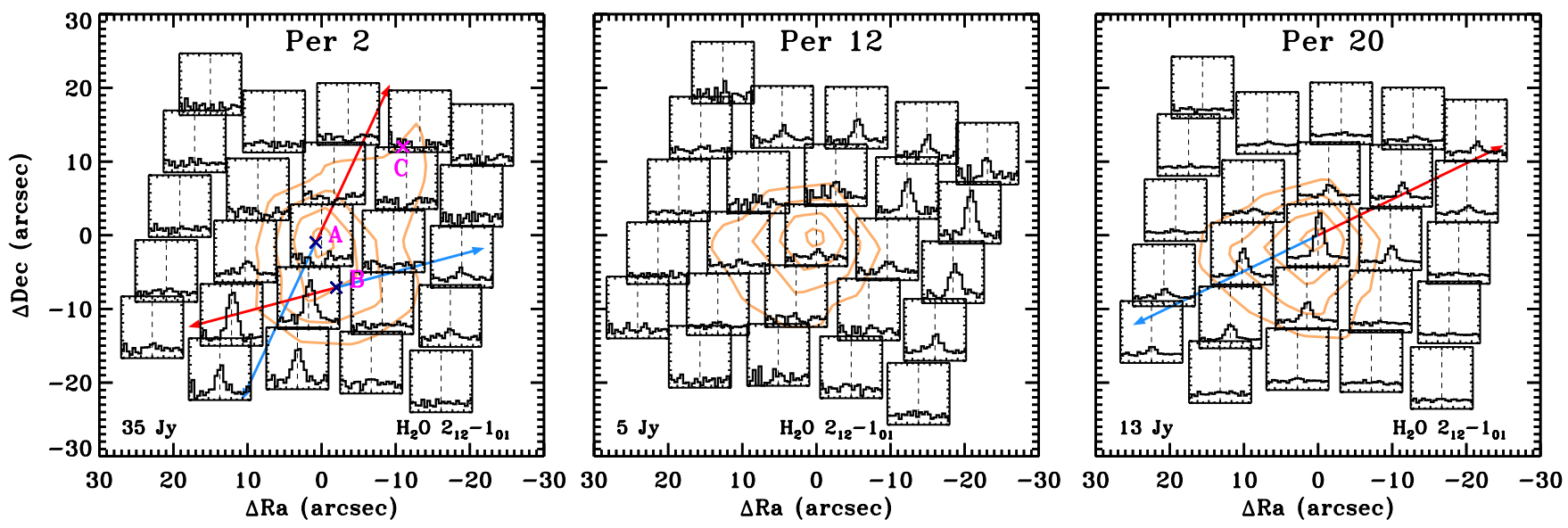

Fig. 1. PACS spectral maps in the $\mathrm{H}_{2} \mathrm{O} 2_{12}-1_{01}$ line at $179 \mu$ m illustrating sources with extended emission due to multiple sources in one field (Per 2), contamination by the outflow driven by another source (Per 12), and associated with the targeted protostar (Per 20). Even though the emission on the maps seems to be extended in many sources in Perseus, the extended emission associated with the targeted protostar itself is detected only towards a few of them (see Table 2). The orange contours show continuum emission at 30\%, 50\%, 70\%, and $90 \%$ of the peak value written in the bottom left corner of each map. L1448 IRS 3A, 3B, and 3C sources and their CO 2-1 outflow directions are shown on the map of Per 2 (Kwon et al. 2006; Looney et al. 2000); the blue outflow lobe of L1448-MM also covers much of the observed field. CO 2-1 outflow directions of Per 20/HH211 are taken from Gueth \& Guilloteau (1999). Wavelengths in microns are translated to the velocity scale on the $X$-axis using laboratory wavelengths (see Table A.1) of the species and cover the range from -600 to $600 \mathrm{~km} \mathrm{~s}^{-1}$. The $Y$-axis shows fluxes in Jy normalized to the spaxel with the brightest line on the map in a range -0.2 to 1.2 .

peaks a few spaxels away from the map center. In both cases, contribution from additional outflows/sources is the cause of the dominant off-source line emission.

To summarize, when the contamination of other sources and their outflows can be excluded, Perseus YSOs show that the $\mathrm{H}_{2} \mathrm{O} 2{ }_{12}-1_{01}$ line emission is either well-confined to the central position on the map or shows at best weak extended line emission (those are marked with "e" in Table 2). In total, 7 out of 22 sources show extended emission in the $\mathrm{H}_{2} \mathrm{O} 2_{12}-1_{01}$ line associated with the targeted sources. Emission in $\mathrm{CO}, \mathrm{OH}$, and other $\mathrm{H}_{2} \mathrm{O}$ lines follows the same pattern (see Fig. A.3). Similarly compact emission was seen in a sample of 30 protostars surveyed in the DIGIT program (Green et al. 2013). In contrast, the WISH PACS survey (Karska et al. 2013) revealed strong extended emission in about half of the 20 low-mass protostars. There, the analysis of patterns of molecular and atomic emission showed that $\mathrm{H}_{2} \mathrm{O}$ and $\mathrm{CO}$ spatially co-exist within the PACS field of view, while $\mathrm{OH}$ and [O I] lines are typically less extended, but also follow each other spatially and not $\mathrm{H}_{2} \mathrm{O}$ and $\mathrm{CO}$.

\subsection{Line detections}

In the majority of our sources, all targeted rotational transitions of $\mathrm{CO}, \mathrm{H}_{2} \mathrm{O}$, and $\mathrm{OH}$ are detected, see Fig. 2 and Tables A.1 and A.3. The [O I] line at $63 \mu \mathrm{m}$ and the [C II] line at $158 \mu \mathrm{m}$ will be discussed separately in a forthcoming paper including all WILL sources (Karska et al., and in prep.) and are not included in the figure and further analysis.

The CO 16-15 line with an upper level energy $\left(E_{\mathrm{u}} / k_{\mathrm{B}}\right)$ of about $750 \mathrm{~K}$ is seen in $17(\sim 80 \%)$, the CO $24-23\left(E_{\mathrm{u}} / k_{\mathrm{B}} \sim\right.$ $1700 \mathrm{~K})$ in $16(\sim 70 \%)$, and the CO $32-31\left(E_{\mathrm{u}} / k_{\mathrm{B}} \sim 3000 \mathrm{~K}\right)$ in $8(\sim 30 \%)$ sources. The most commonly detected ortho$\mathrm{H}_{2} \mathrm{O}$ lines are: the $2_{12}-1_{01}$ line at $179 \mu \mathrm{m}\left(E_{\mathrm{u}} / k_{\mathrm{B}} \sim 110 \mathrm{~K}\right)$ and the $4_{04}-3_{13}$ line at $125 \mu \mathrm{m}\left(E_{\mathrm{u}} / k_{\mathrm{B}} \sim 320 \mathrm{~K}\right)$, seen in 15 sources ( $\sim 70 \%)$, whereas the $6_{16}-5_{05}$ line at $82 \mu \mathrm{m}\left(E_{\mathrm{u}} / k_{\mathrm{B}} \sim 640 \mathrm{~K}\right)$ is detected in 13 sources $(\sim 60 \%)$. The para- $\mathrm{H}_{2} \mathrm{O}$ line $33_{22}-2_{11}$ at $90 \mu \mathrm{m}\left(E_{\mathrm{u}} / k_{\mathrm{B}} \sim 300 \mathrm{~K}\right)$ is seen toward 9 sources $(\sim 50 \%)$.
Three $\mathrm{OH}$ doublets targeted as part of the WILL survey, the $\mathrm{OH}^{2} \Pi_{1 / 2}, J=1 / 2-{ }^{2} \Pi_{3 / 2}, J=3 / 2$ at $79 \mu \mathrm{m},{ }^{2} \Pi_{3 / 2} J=7 / 2-5 / 2$ doublet at $84 \mu \mathrm{m}\left(E_{\mathrm{u}} / k_{\mathrm{B}} \sim 290 \mathrm{~K}\right)$, and ${ }^{2} \prod_{1 / 2} J=3 / 2-1 / 2$ doublet at $163 \mu \mathrm{m}\left(E_{\mathrm{u}} / k_{\mathrm{B}} \sim 270 \mathrm{~K}\right)$, are detected in 14,15 , and 13 objects, respectively $(\sim 60-70 \%)$.

Sources without any detections of molecular lines associated with the targeted protostars are Per 4, 7, 12, and 17 (see Table A.3). In Per 13 only two weak $\mathrm{H}_{2} \mathrm{O}$ lines at 108 and $125 \mu \mathrm{m}$ are seen, whereas in Per 16 only a few of the lowest- $J$ $\mathrm{CO}$ lines are detected. A common characteristic of this weakline group of objects, is a low bolometric temperature (all except Per 4) and a low bolometric luminosity (see Table 1), always below $1.3 L_{\odot}$. However, our sample also includes a few objects with similarly low values of $L_{\text {bol }}$, that show many more molecular lines (in particular Per 3, but also Per 15 and 21), so low luminosity by itself is not a criterion for weak lines. On the other hand, low bolometric temperature and high luminosity is typically connected with strong line emission (Kristensen et al. 2012; Karska et al. 2013).

\subsection{Observed line ratios}

Observed line ratios are calculated using the fluxes obtained from the entire $5 \times 5$ PACS maps in cases of extended emission where contamination by a nearby source and/or outflows is excluded. For point sources, a wavelength-dependent PSF correction factor is applied to fluxes obtained from the central spaxel (for details, see Sect. 2). In principle, ratios using lines that are close in wavelength could be calculated using smaller flux extraction regions, and no PSF correction would be required. However, the transition wavelengths of $\mathrm{H}_{2} \mathrm{O}$ are not proportional to the upper energy levels, as is the case for $\mathrm{CO}$, and comparisons of lines tracing similar gas have to rely on lines that lie far apart in wavelength. We explore to what extent the size of the extraction region affects the inferred line ratios. Since our aim is to understand the influence of the extended emission associated with the source(s), the ratios of lines close in wavelength are studied to avoid the confusion caused by the PSF variations. 


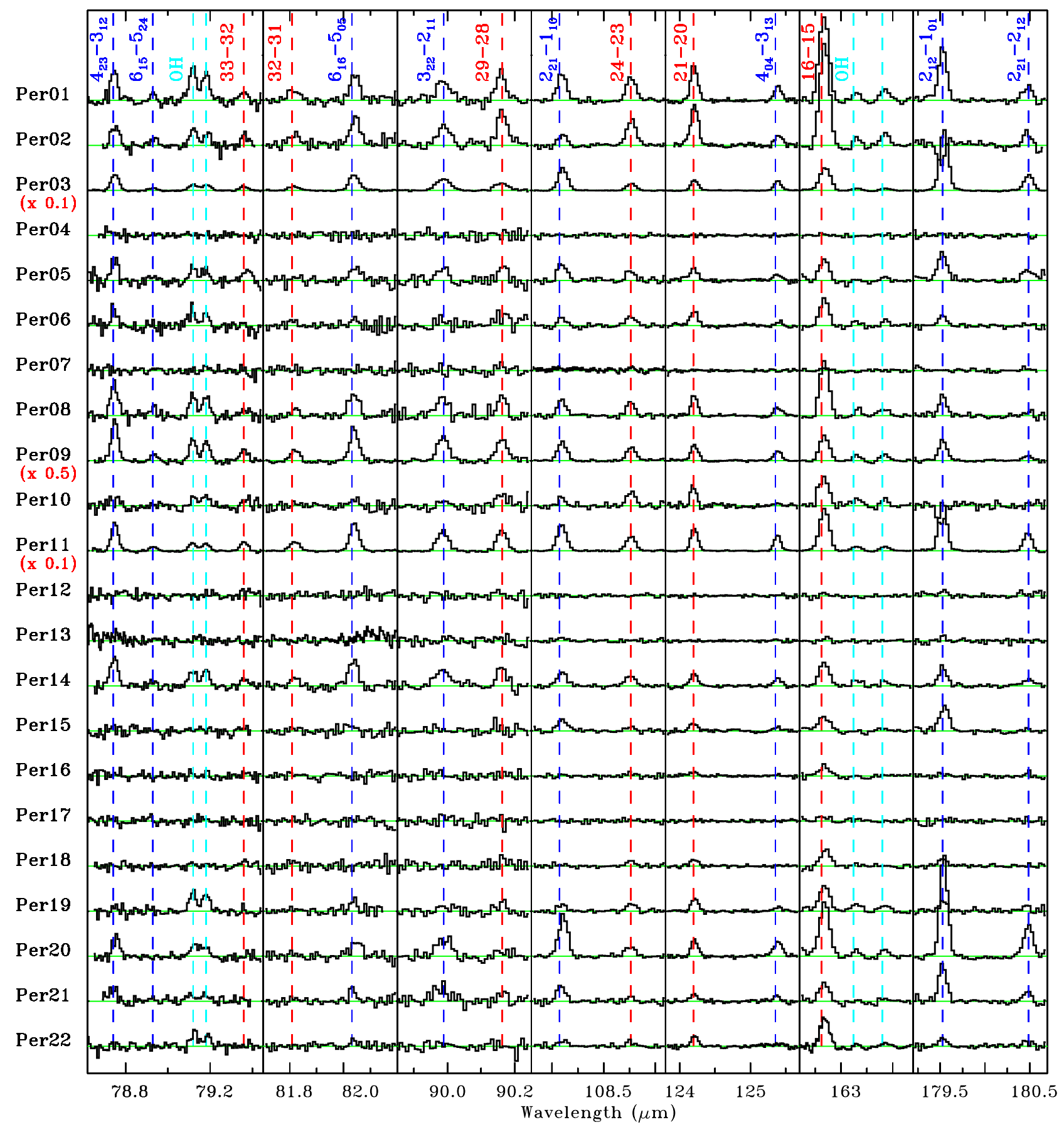

Fig. 2. Line survey of deeply-embedded young stellar objects in Perseus at the central position on the maps. Spectra are continuum subtracted and not corrected for the PSF. Line identification of $\mathrm{CO}$ (red), $\mathrm{H}_{2} \mathrm{O}$ (blue), and $\mathrm{OH}$ (light blue), are shown. Each spectrum is on a scale from 0 to $5 \mathrm{Jy}$ in the $y$-axis, with the brightest sources - Per 3, Per 9, and Per 11 - scaled down in flux density by a factor of $0.1,0.5$, and 0.1 , respectively.

Fluxes of the nearby $\mathrm{H}_{2} \mathrm{O} 4_{04}-313$ and $\mathrm{CO} 21-20$ lines located at $124-125 \mu \mathrm{m}$ are calculated first using only the central spaxel (compact region) and then using all the spaxels with detected line emission (extended region). Figure 3 illustrates that the line ratios calculated in these two regions are fully consistent, both for sources with compact and extended emission. Therefore, for the subsequent analysis the extended region is used for comparisons with models. Table A.4 shows the minimum and maximum values of the observed line ratios, their mean values, and the standard deviations for all sources with detections.
Even taking into account the uncertainties in flux extraction, the line ratios span remarkably narrow ranges of values, see Table 3 for a selection of $\mathrm{H}_{2} \mathrm{O}, \mathrm{CO}$, and $\mathrm{OH}$ line ratios. The largest range is seen in the $\mathrm{H}_{2} \mathrm{O} 2_{12}-1_{01} / \mathrm{CO} 16-15$ line ratio, which spans an order of magnitude. In all the other cases the line ratios are similar up to a factor of a few. The most similar are the $\mathrm{OH}$ line ratios which differ only by a factor of two, consistent with previous studies based on a large sample of lowmass YSOs in Wampfler et al. (2013). The observed similarities also imply that the line ratios do not depend on protostellar luminosity, bolometric temperature, or envelope mass (see Fig. B.1). 
Table 3. Comparison of observed $\mathrm{H}_{2} \mathrm{O}, \mathrm{CO}$, and $\mathrm{OH}$ line ratios with literature values.

\begin{tabular}{|c|c|c|c|c|c|c|c|}
\hline Object & $22_{12}-1_{01} / 16-15$ & $4_{04}-3_{13} / 24-23$ & $16-15 / 24-23$ & $2_{12}-1_{01} / 4_{04}-3_{13}$ & $22_{21}-1_{10} / 4_{04}-3_{13}$ & $\mathrm{OH} 84 / 79$ & Ref. \\
\hline \multicolumn{8}{|c|}{ Perseus (this work) } \\
\hline Perseus & $0.2-2.4$ & $0.2-1.1$ & $1.2-4.6$ & $1.3-6.3$ & $1.4-5.5$ & $1.1-2.4$ & This work \\
\hline \multicolumn{8}{|c|}{ On-source (literature) } \\
\hline SMM3 b & $1.0 \pm 0.1$ & $0.5 \pm 0.2$ & $3.3 \pm 0.8$ & $7.1 \pm 1.0$ & $3.7 \pm 1.0$ & $1.4 \pm 0.7$ & (1) \\
\hline $\mathrm{c}$ & $0.9 \pm 0.2$ & $0.9 \pm 0.3$ & $4.6 \pm 1.0$ & $4.9 \pm 1.0$ & $2.3 \pm 0.8$ & $2.4 \pm 1.2$ & (1) \\
\hline r & $1.0 \pm 0.1$ & $2.0 \pm 0.7$ & $9.4 \pm 2.3$ & $4.7 \pm 1.0$ & $3.1 \pm 0.9$ & n.d. & (1) \\
\hline SMM4 r & $0.8 \pm 0.1$ & $0.4 \pm 0.1$ & $3.6 \pm 0.6$ & $7.8 \pm 1.6$ & $3.0 \pm 1.3$ & $1.1 \pm 0.5$ & (1) \\
\hline L1448-MM & $2.3 \pm 1.3$ & $1.2 \pm 0.7$ & $2.1 \pm 1.2$ & $4.1 \pm 2.3$ & $2.6 \pm 1.5$ & $1.4 \pm 0.9$ & (2) \\
\hline NGC 1333 I4B & $1.0 \pm 0.1$ & $1.0 \pm 0.1$ & $1.9 \pm 0.1$ & $1.9 \pm 0.1$ & $2.0 \pm 0.1$ & $1.2 \pm 0.2$ & (3) \\
\hline \multicolumn{8}{|c|}{ Off-source (literature) } \\
\hline L1157 B1 & $4.0 \pm 0.5$ & - & - & 11.0 & $2.9 \pm 1.2$ & n.d. & $(4,5)$ \\
\hline $\mathrm{B} 1^{\prime}$ & $2.1 \pm 0.2$ & - & - & $9.2 \pm 2.2$ & $2.8 \pm 1.0$ & $0.9 \pm 0.5$ & $(4,5)$ \\
\hline B2 & $17.0 \pm 8.4$ & n.d. & $>0.3$ & $>7.7$ & n.d. & - & (6) \\
\hline $\mathrm{R}$ & $5.3 \pm 2.4$ & $>0.26$ & $>0.5$ & $10.6 \pm 4.7$ & $1.8 \pm 0.8$ & - & (6) \\
\hline L1448 B2 & $2.3 \pm 0.4$ & $0.4 \pm 0.3$ & $2.8 \pm 0.9$ & $15.0 \pm 6.5$ & $3.7 \pm 1.6$ & - & (6) \\
\hline R4 & $8.2 \pm 3.0$ & $>0.3$ & $>0.9$ & $23.2 \pm 9.7$ & $3.3 \pm 1.2$ & - & (6) \\
\hline
\end{tabular}

Notes. Sources in the upper part of the table refer to protostellar positions within the PACS maps, sources in the lower part refer to shock positions away from the protostar. Ranges of line ratios calculated for Perseus sources are listed at the top. Line ratios of sources that exceed the Perseus values are shown in boldface. The L1157 B1' position refers to the high-excitation $\mathrm{CO}$ emission peak close to the nominal position of the B1 shock spot (Benedettini et al. 2012). Non-detections are abbreviated with n.d. OH 84/79 refers to the ratio of two $\mathrm{OH}$ doublets, at 84 and $79 \mu \mathrm{m}$, respectively. The total flux of the $84 \mu \mathrm{m}$ doublet is calculated by multiplying the $84.6 \mu \mathrm{m}$ flux by two, because of the blending of the $84.4 \mu \mathrm{m}$ line with the CO 31-30 line.

References. (1) Dionatos et al. (2013); (2) Lee et al. (2013); (3) Herczeg et al. (2012); (4) Benedettini et al. (2012); (5) Busquet et al. (2014); (6) Santangelo et al. (2013).

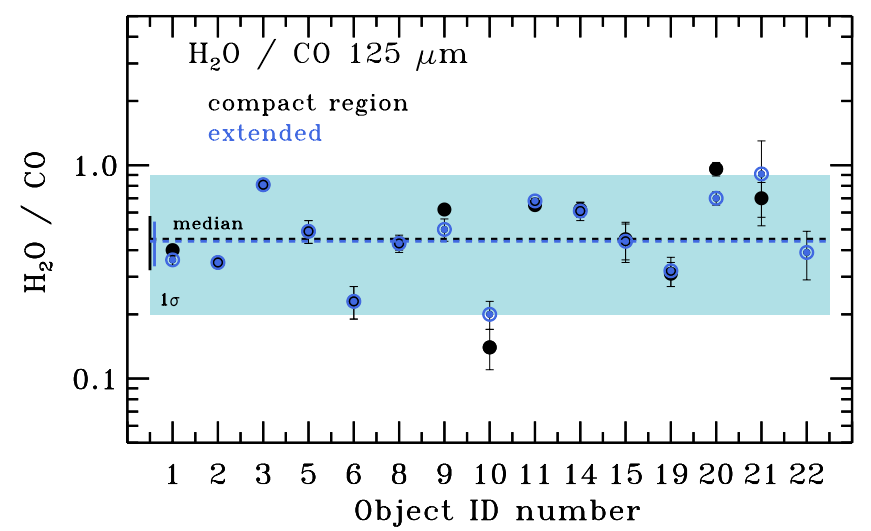

Fig. 3. Flux ratios of the $\mathrm{H}_{2} \mathrm{O} 4_{04}-3_{13}$ and $\mathrm{CO} 21-20$ lines at $\sim 125 \mu \mathrm{m}$ calculated using compact and extended flux extraction regions (see text) corrected for contamination from other sources/outflows. Median values for the two configurations are shown by the dashed line. The light blue rectangle shows the parameter space between the minimum and maximum values of line ratios in the more extended configuration. The error bars reflect the uncertainties in the measured fluxes of the two lines, excluding the calibration error, which is the same for those closely spaced lines.

Our line ratios for Perseus sources are consistent with the previously reported values for other deeply-embedded protostars observed in the same way ("on source") as tabulated in Table 3. Some differences are found for PACS observations of shock positions away from the protostar ("off source"). Most notably, the ratios using the low excitation $\mathrm{H}_{2} \mathrm{O} 2_{12}-1_{01}$ line at $179 \mu \mathrm{m}$ are up to a factor of two larger than those observed in the protostellar vicinity. Such differences are not seen when more highlyexcited $\mathrm{H}_{2} \mathrm{O}$ lines are compared with each other, for example $\mathrm{H}_{2} \mathrm{O} 2_{21}-1_{10}$ and $4_{04}-3_{13}$ lines, or with the high- $J \mathrm{CO}$ lines, for example CO 24-23. The ratios of two CO lines observed away from the protostar, e.g., the $\mathrm{CO} 16-15$ and $\mathrm{CO} 24-23$ ratios, are at the low end of the range observed toward the protostellar position.

Spectrally-resolved profiles of the $\mathrm{H}_{2} \mathrm{O} \quad 2_{12}-1_{01}$ line observed with HIFI toward the protostar position reveal absorptions at source velocity removing about $10 \%$ of total line flux (e.g., Kristensen et al. 2010; Mottram et al. 2014). Our unresolved PACS observations therefore provide a lower limit to the $\mathrm{H}_{2} \mathrm{O}$ emission in the $2_{12}-1_{01}$ line. This effect, however, is too small to explain the differences in the line ratios at the "on source" and "off source" positions.

\section{Analysis}

Multiple molecular transitions over a wide range of excitation energies are detected, which points to the presence of hot, dense gas and can be used to constrain the signatures of shocks created as a result of outflow-envelope interaction. In particular, the line ratios of $\mathrm{H}_{2} \mathrm{O}, \mathrm{CO}$, and $\mathrm{OH}$ are useful probes of various shock types and parameters that do not suffer from distance uncertainties.

Similarities between the spatial extent of different molecules (Sect. 3.1) coupled with similarities in the velocity-resolved line profiles among these species (Kristensen et al. 2010; Y1ld1z et al. 2013; San José-García et al. 2013; Mottram et al. 2014) strongly suggest that all highly-excited lines of $\mathrm{CO}$ and $\mathrm{H}_{2} \mathrm{O}$ arise from the same gas. Some differences may occur for $\mathrm{OH}$, which is also associated with dissociative shocks and can be affected by radiative excitation (see Sect. 5). Modeling of absolute line fluxes requires sophisticated two-dimensional (2D) physical source models for the proper treatment of the beam filling factor (Visser et al. 2012). Those models also show that UV heating alone is not sufficient to account for the high excitation lines. Hence, the focus in this analysis is on shocks. Since the absolute flux depends sensitively on the assumed emitting area, in the subsequent analysis only the line ratios are compared.

In the following sections, properties of shock models and the predicted line emission in various species are discussed 
(Sect. 4.1) and observations are compared with the models, using line ratios of the same species (Sect. 4.2), and different species (Sect. 4.3). Special focus will be given to $C$-type shocks where grids of model results are available in the literature. Observations suggest that most of the mass of hot gas is in $C$-type shocks toward the central protostellar positions, at least for $\mathrm{H}_{2} \mathrm{O}$ and CO with $J<30$ (Kristensen et al. 2013, and in prep.); higher$J \mathrm{CO}, \mathrm{OH}$ and $[\mathrm{O} \mathrm{I}]$ transitions, on the other hand, will primarily trace $J$-type shocks (e.g., Wampfler et al. 2013; Kristensen et al. 2013). The excitation of $\mathrm{OH}$ and [O I] will be analyzed in a forthcoming paper; the $J>30 \mathrm{CO}$ emission is only detected toward $\sim 30 \%$ of all sources and so is likely unimportant for the analysis and interpretation of the data presented here. Only a limited discussion of $J$-type shocks is therefore presented below.

\subsection{Model line emission}

Models of shocks occurring in a medium with physical conditions typical for the envelopes of deeply-embedded young stellar objects provide a valuable tool for investigating shock characteristics: shock type, velocity, and the pre-shock density of (envelope) material.

Model grids have been published using a simple 1D geometry either for steady-state $C$ and $J$ type shocks (Hollenbach et al. 1989; Kaufman \& Neufeld 1996; Flower \& Pineau des Forêts 2010) or time-dependent $C-J$ type shocks (Gusdorf et al. 2008, 2011; Flower \& Pineau des Forêts 2012). The latter are nonstationary shocks, where a $J$-type front is embedded in a $C$-type shock (Chieze et al. 1998; Lesaffre et al. 2004a,b). These shocks are intermediate between pure $C$ - and $J$-type shocks and have temperatures and physical extents in between the two extremes.

$C$ - $J$ shocks may be required for the youngest outflows with ages less than $10^{3}$ yrs, (Flower \& Pineau des Forêts 2012, 2013, for the case of IRAS4B, Per 11). Here the dynamical age of the outflow is taken as an upper limit of that of the shock itself, which may be caused by a more recent impact of the wind on the envelope. The age of our sources is on the order of $10^{5} \mathrm{yrs}$ (Sadavoy et al. 2014, for Class 0 sources in Perseus) and they should have been driving winds and jets for the bulk of this period, so this timescale is long enough for any shocks close to the source position to have reached steady state. While we cannot exclude that a few individual shocks have been truncated, our primary goal is to examine trends across the sample. Invoking $C$ - $J$ type shocks with a single truncation age as an additional free parameter is therefore not a proper approach for this study. The focus is therefore placed on comparing $C$-type shock results from Kaufman \& Neufeld (1996, KN96 from now on) and Flower \& Pineau des Forêts (2010, F+PdF10 from now on) with the observations.

All models assume the same initial atomic abundances and similarly low degrees of ionization, $x_{\mathrm{i}} \sim 10^{-7}$ for $C$ shocks. The pre-shock transverse magnetic field strength is parametrized as $B_{0}=b \times \sqrt{n_{\mathrm{H}}\left(\mathrm{cm}^{-3}\right)} \mu$ gauss, where $n_{\mathrm{H}}$ is the pre-shock number density of atomic hydrogen and $b$ is the magnetic scaling factor, which is typically $0.1-3$ in the ISM (Draine 1980). The value of $b$ in KN96 and F+PdF10 is fixed at a value of 1 .

The main difference between the two shock models is the inclusion of grains in the F+PdF10 models (Flower \& Pineau des Forêts 2003). The latter models assume a standard MRN distribution of grain sizes (Mathis et al. 1977) for grain radii between $0.01 \mu \mathrm{m}$ and $0.3 \mu \mathrm{m}$ and a fractional abundance of the PAH in the gas phase of $10^{-6}$ (the role of PAHs and the sizes of grains are discussed in Flower \& Pineau des Forêts 2003, 2012). As the electrons are accelerated in the magnetic precursor, they attach themselves to grains thereby charging the grains and thus increasing the density of the ionized fluid significantly. This increase in density has the effect of enhancing the ion-neutral coupling (Draine 1980), thereby effectively lowering the value of $b$ compared to the KN96 models. As a consequence, the maximum kinetic temperature is higher in the $\mathrm{F}+\mathrm{PdF} 10$ models for a given shock velocity, $v$. The stronger coupling between the ions and neutrals results in narrower shocks (Flower \& Pineau des Forêts 2010), with shock widths scaling as $\propto b^{2}\left(x_{\mathrm{i}} n_{\mathrm{H}} v\right)^{-1}$ (Draine 1980). This proportionality does not capture the ion-neutral coupling exactly, as, for example, the grain size distribution influences the coupling (Guillet et al. 2007, 2011). The compression in $C$ shocks also changes with the coupling since the post-shock density depends on the magnetic field, $n_{\text {post }} \sim 0.8 v n_{\mathrm{H}} b^{-1}$ (e.g., Karska et al. 2013). The column density of emitting molecules is a function of both shock width and compression factor, and as a zeroth-order approximation the column density is $N \sim n_{\text {post }} \times L \sim b x_{\mathrm{i}}^{-1}$. The ionization degree is not significantly different between the KN96 and F+PdF10 models because it is primarily set by the cosmic ray ionization rate ( $\left.\zeta=5 \times 10^{-17} \mathrm{~s}^{-1}, \mathrm{~F}+\mathrm{PdF} 10\right)$, and thus the F+PdF10 models predict lower column densities than the KN96 models for a given velocity and density.

Another important difference is that the $\mathrm{F}+\mathrm{PdF} 10$ models take into account that molecules frozen out onto grain mantles can be released through sputtering when the shock velocity exceeds $\sim 15 \mathrm{~km} \mathrm{~s}^{-1}$ (Flower \& Pineau des Forêts 2010, 2012; Van Loo et al. 2013). Therefore, the gas-phase column densities of molecules locked up in ices increase above this threshold shock velocity with respect to the KN96 models, an effect which applies to both $\mathrm{CO}$ and $\mathrm{H}_{2} \mathrm{O}$. Furthermore, $\mathrm{H}_{2} \mathrm{O}$ forms more abundantly in the post-shock gas of F+PdF10 models, because $\mathrm{H}_{2}$ reformation is included, unlike in the KN96 models (Flower \& Pineau des Forêts 2010).

Molecular emission is tabulated by KN96 for a wide range of shock velocities, from $v=5$ to $45 \mathrm{~km} \mathrm{~s}^{-1}$ in steps of $5 \mathrm{~km} \mathrm{~s}^{-1}$, and a wide range of pre-shock densities $n_{\mathrm{H}}$, from $10^{4}$ to $10^{6.5} \mathrm{~cm}^{-3}$ in steps of $10^{0.5} \mathrm{~cm}^{-3}$. The F+PdF10 grid is more limited in size, providing line intensities for only two values of pre-shock densities, namely $10^{4}$ and $10^{5} \mathrm{~cm}^{-3}$, and a comparable range of shock velocities, but calculated in steps of $10 \mathrm{~km} \mathrm{~s}^{-1}$. Calculations are provided for $\mathrm{CO}$ transitions from $J=1-0$ to $J=60-59$ in KN96 and only up to $J=20-19$ in $\mathrm{F}+\mathrm{PdF} 10$. The two sets of models use different collisional rate coefficients to calculate the $\mathrm{CO}$ excitation. $\mathrm{F}+\mathrm{PdF} 10$ show line intensities for many more $\mathrm{H}_{2} \mathrm{O}$ transitions (in total $\sim 120$ lines in the PACS range, see Sect. 2) than in the older KN96 grid (18 lines in the same range), which was intended for comparisons with the Submillimeter Wave Astronomy Satellite (SWAS, Melnick et al. 2000) and ISO data. KN96 use collisional excitation rates for $\mathrm{H}_{2} \mathrm{O}$ from Green et al. (1993) and $\mathrm{F}+\mathrm{PdF} 10$ from Faure et al. (2007). Line intensities for $\mathrm{OH}$ are only computed by KN96, assuming only collisional excitation and using the oxygen chemical network of Wagner \& Graff (1987). The reaction rate coefficients in that network are within a factor of 2 of the newer values by Baulch et al. (1992) and tabulated in the UMIST database (www . udfa . net, McElroy et al. 2013), see also a discussion in van Dishoeck et al. (2013).

We also use $\mathrm{CO}$ fluxes extracted from the grid of models presented by Kristensen et al. (2007), since high- $J$ CO lines are missing in $\mathrm{F}+\mathrm{PdF} 10$. This grid is based on the shock model presented in Flower \& Pineau des Forêts (2003) and covers densities from $10^{4}-10^{7} \mathrm{~cm}^{-3}$ and velocities from $10-50 \mathrm{~km} \mathrm{~s}^{-1}$ (denoted as $\mathrm{F}+\mathrm{PdF}^{*}$ from now on). The main difference compared 
to the results from $\mathrm{F}+\mathrm{PdF} 10$ is that $\mathrm{CO}$ and $\mathrm{H}_{2} \mathrm{O}$ level populations are not calculated explicitly through the shock; instead analytical cooling functions are used to estimate the relevant line cooling and afterwards are line fluxes extracted (Flower \& Gusdorf 2009). Models with $b=1$ are used. The CO line fluxes presented here are computed using the 3D non-LTE radiative transfer code LIME (Brinch \& Hogerheijde 2010), for levels up to $J=80-79$. The $\mathrm{CO}$ collisional rate coefficients from Yang et al. (2010) extended by Neufeld (2012) are used.

In the following sections, the model fluxes of selected $\mathrm{CO}$, $\mathrm{H}_{2} \mathrm{O}$, and $\mathrm{OH}$ lines are discussed for a range of shock velocities and three values of pre-shock densities: $10^{4}, 10^{5}$, and $10^{6} \mathrm{~cm}^{-3}$. We note that the post-shock densities traced by observations are related to the pre-shock densities via the compression factor dependent on the shock velocity and magnetic field. In $C$ shocks, the compression factor is about 10 (e.g., Karska et al. 2013).

Figure 4 compares model fluxes of various $\mathrm{CO}, \mathrm{OH}$, and $\mathrm{H}_{2} \mathrm{O}$ lines from the KN96 models (panels a and d) and, for a few selected $\mathrm{CO}$ and $\mathrm{H}_{2} \mathrm{O}$ lines, compares the results with the $\mathrm{F}+\mathrm{PdF} 10$ or $\mathrm{F}+\mathrm{PdF}^{*}$ models (panels b, c, e, and f).

\subsubsection{CO}

The KN96 model line fluxes for CO 16-15, CO 21-20, and CO 29-28 are shown in panel a of Fig. 4. The upper energy levels of these transitions lie at $750 \mathrm{~K}, 1280 \mathrm{~K}$, and $2900 \mathrm{~K}$, respectively, while with increasing shock velocity, the peak $C$-shock temperature increases from about $400 \mathrm{~K}$ to $3200 \mathrm{~K}$ (for 10 to $40 \mathrm{~km} \mathrm{~s}^{-1}$ ) and is only weakly dependent on the assumed density (see Fig. 3 of KN96). Therefore, the CO 16-15 line is already excited at relatively low shock velocities $\left(v \sim 10 \mathrm{~km} \mathrm{~s}^{-1}\right.$, for $n_{\mathrm{H}}=10^{4} \mathrm{~cm}^{-3}$ ), whereas the higher- $J$ levels become populated at higher velocities. At a given shock velocity, emission from the CO 16-15 line is the strongest because of its lower critical density, $n_{\mathrm{cr}} \sim 9 \times 10^{5} \mathrm{~cm}^{-3}$ at $T=1000 \mathrm{~K}$ (Neufeld 2012). This situation only changes for the highest pre-shock densities, when the line becomes thermalized and the cooling in other lines dominates.

The CO 16-15 flux from the F+PdF10 $C$-type shock models is comparable to the KN96 flux for the $v \sim 10 \mathrm{~km} \mathrm{~s}^{-1}$ shock, but increases less rapidly with shock velocity despite the sputtering from grain mantles (panel b of Fig. 4). Because of the lower magnetic scaling factor $b$ in the models with grains (see Sect. 4.1), it is expected that the column density and the corresponding line fluxes are lower in the F+PdF10 models. For slow shocks the higher temperatures in the latter models compensate for the smaller column density resulting in a similar CO 16-15 flux.

In $J$-type shocks, the peak temperatures of the post-shock gas are 1400, 5500, 12000 , and $22000 \mathrm{~K}$ for the shock velocities of $10,20,30$, and $40 \mathrm{~km} \mathrm{~s}^{-1}$ respectively (Neufeld \& Dalgarno 1989; Kaufman \& Neufeld 1996). For $10-20 \mathrm{~km} \mathrm{~s}^{-1}$ shocks, these high temperatures more easily excite the CO 16-15 line with respect to $C$-type shock emission. For shock velocities above $20 \mathrm{~km} \mathrm{~s}^{-1}$, such high temperatures can lead to the collisional dissociation of $\mathrm{H}_{2}$ and subsequent destruction of $\mathrm{CO}$ and $\mathrm{H}_{2} \mathrm{O}$ molecules, resulting in the decrease in $\mathrm{CO}$ fluxes. This effect requires high densities and therefore the $\mathrm{CO}$ flux decrease is particularly strong for the pre-shock densities $10^{5} \mathrm{~cm}^{-3}$.

\subsection{2. $\mathrm{H}_{2} \mathrm{O}$}

The $\mathrm{H}_{2} \mathrm{O}$ fluxes show a strong increase with shock velocities above $v \sim 10-15 \mathrm{~km} \mathrm{~s}^{-1}$ in both models, especially at low pre-shock densities (panels d-f of Fig. 4). At this velocity, the
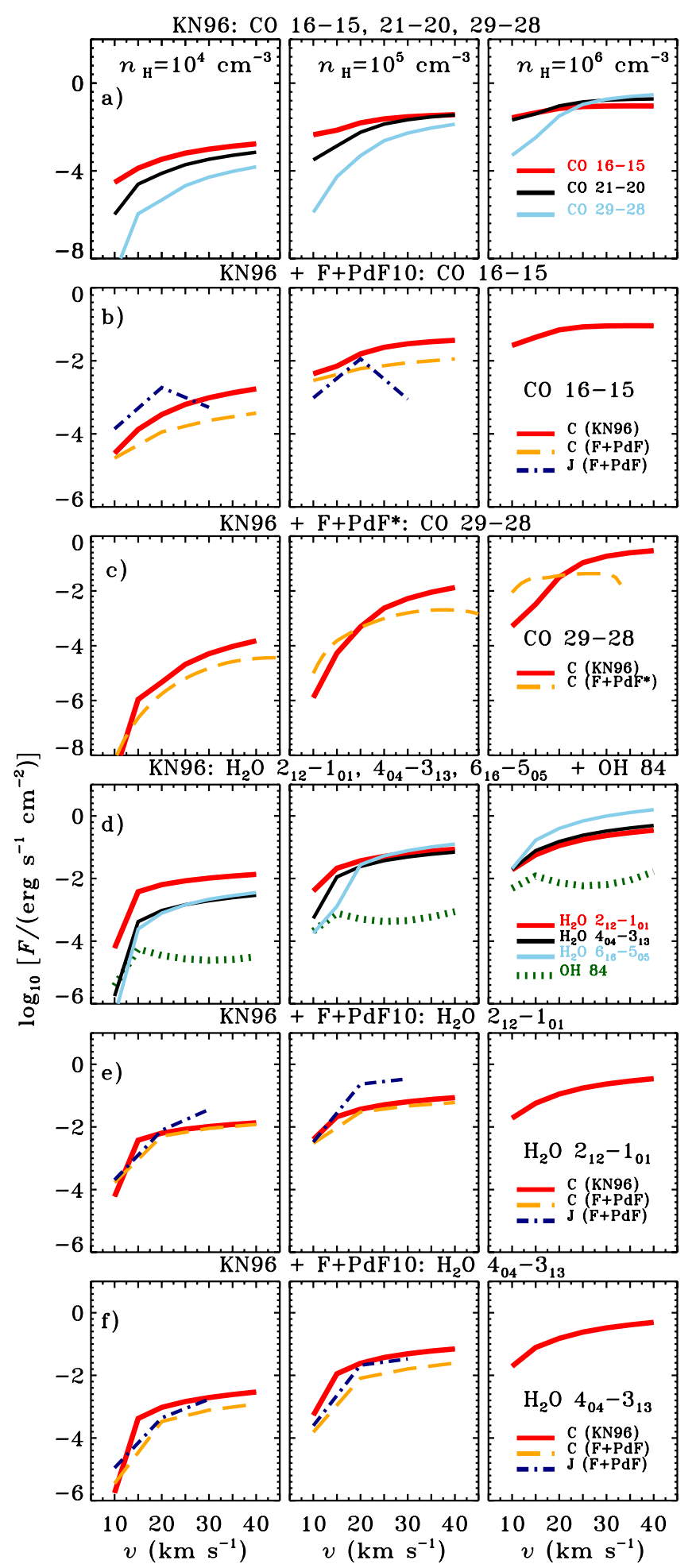

Fig. 4. Absolute fluxes of selected $\mathrm{CO}, \mathrm{H}_{2} \mathrm{O}$, and $\mathrm{OH}$ lines predicted by Kaufman \& Neufeld and Flower \& Pineau des Forêts models and shown as a function of shock velocity and for pre-shock densities of $10^{4} \mathrm{~cm}^{-3}$ (left), $10^{5} \mathrm{~cm}^{-3}$ (center), and $10^{6} \mathrm{~cm}^{-3}$ (right). The latter models are available only for the pre-shock densities of $10^{4} \mathrm{~cm}^{-3}$ and $10^{5} \mathrm{~cm}^{-3}$. $\mathrm{OH} 84$ refers to the $\mathrm{OH}^{2} \Pi_{3 / 2} J=7 / 2-5 / 2$ doublet at $84 \mu \mathrm{m}$.

gas temperature exceeds $\sim 400 \mathrm{~K}$, so the high-temperature route of $\mathrm{H}_{2} \mathrm{O}$ formation becomes efficient (Elitzur \& Watson 1978; Elitzur \& de Jong 1978; Bergin et al. 1998; KN96), which 
quickly transfers all gas-phase oxygen into $\mathrm{H}_{2} \mathrm{O}$ via reactions with $\mathrm{H}_{2}$ (KN96).

In contrast to $\mathrm{CO}$, the upper level energies of the observed $\mathrm{H}_{2} \mathrm{O}$ lines are low and cover a narrow range of values, $E_{\text {up }} \sim$ 200-600 K. As a result, the effect of peak gas temperature on the $\mathrm{H}_{2} \mathrm{O}$ excitation is less pronounced (Fig. 3 of KN96) and after the initial increase with shock velocity, the $\mathrm{H}_{2} \mathrm{O}$ fluxes in the KN96 models stay constant for all lines. For high pre-shock densities, the higher lying levels are more easily excited and, as a consequence, the fluxes of the $\mathrm{H}_{2} \mathrm{O} 6_{16}-5_{05}$ line become larger than those of the $\mathrm{H}_{2} \mathrm{O} 2_{12}-1_{01}$ line. The critical densities of these transitions are about two orders of magnitude higher than for the CO 16-15 line and the levels are still sub-thermally excited at densities of $10^{6}-10^{7} \mathrm{~cm}^{-3}$ (and effectively optically thin, Mottram et al. 2014).

The $\mathrm{H}_{2} \mathrm{O} 2{ }_{12}-1_{01}$ fluxes in the $C$-type $\mathrm{F}+\mathrm{PdF} 10$ models are remarkably similar to those found by KN96 (panel e of Fig. 4), while the $\mathrm{H}_{2} \mathrm{O} 4_{04}-3_{13}$ fluxes are lower by a factor of a few over the full range of shock velocities and pre-shock densities in the F+PdF10 models (panel $\mathrm{f}$ of Fig. 4). Lower $\mathrm{H}_{2} \mathrm{O}$ fluxes are expected because of the smaller column of $\mathrm{H}_{2} \mathrm{O}$ in the models with grains (Sect. 4.1). For the lower- $J$ lines, the various factors (lower column density through a shock but inclusion of ice sputtering and $\mathrm{H}_{2}$ reformation) apparently conspire to give similar fluxes as for KN96.

In $J$-type shocks, the fluxes of $\mathrm{H}_{2} \mathrm{O} \quad 2{ }_{12}-1_{01}$ and $4_{04}-3_{13}$ lines increase sharply for shock velocities $10-20 \mathrm{~km} \mathrm{~s}^{-1}$ (panels e and $\mathrm{f}$ of Fig. 4). The increase is not as steep at $30 \mathrm{~km} \mathrm{~s}^{-1}$ shocks for $n_{\mathrm{H}}=10^{5} \mathrm{~cm}^{-3}$, when the collisional dissociation of $\mathrm{H}_{2}$ and subsequent destruction of $\mathrm{H}_{2} \mathrm{O}$ molecules occurs (fluxes for larger shock velocities are not computed in $\mathrm{F}+\mathrm{PdF} 10$ and hence not shown). Below $30 \mathrm{~km} \mathrm{~s}^{-1}$, line fluxes from $J$-type shocks are comparable to those from $C$-type shock predictions except for the $\mathrm{H}_{2} \mathrm{O} 2_{12}-1_{01}$ fluxes at high pre-shock densities, which are an order of magnitude higher with respect to the $C$-type shock predictions. The difference could be due to smaller opacities for the low-excitation $\mathrm{H}_{2} \mathrm{O}$ line in the $J$ shocks.

\subsection{3. $\mathrm{OH}$}

The fluxes of the ${ }^{2} \prod_{3 / 2} J=7 / 2-5 / 2$ doublet at $84 \mu \mathrm{m}\left(E_{\mathrm{u}} / k_{\mathrm{B}} \sim\right.$ $290 \mathrm{~K})$ calculated with the KN96 models are shown with the $\mathrm{H}_{2} \mathrm{O}$ lines in panel d of Fig. 4. Not much variation is seen as a function of shock velocity, in particular beyond the initial increase from 10 to $15 \mathrm{~km} \mathrm{~s}^{-1}$, needed to drive oxygen to $\mathrm{OH}$ by the reaction with $\mathrm{H}_{2}$. At about $15 \mathrm{~km} \mathrm{~s}^{-1}$, the temperature is high enough to start further reactions with $\mathrm{H}_{2}$ leading to $\mathrm{H}_{2} \mathrm{O}$ production. The trend with increasing pre-shock density is more apparent, with $\mathrm{OH}$ fluxes increasing by two orders of magnitude between the $10^{4} \mathrm{~cm}^{-3}$ to $10^{6} \mathrm{~cm}^{-3}$, as the density becomes closer to the critical density of the transition.

\subsection{Models versus observations - line ratios of the same species}

Comparison of observed and modeled line ratios of different pairs of $\mathrm{CO}, \mathrm{H}_{2} \mathrm{O}$, and $\mathrm{OH}$ transitions is shown in Fig. 5. The line ratios are a useful probe of molecular excitation and therefore can be used to test whether the excitation in the models is reproduced correctly, which in turn depends on density and temperature, and thus shock velocity.
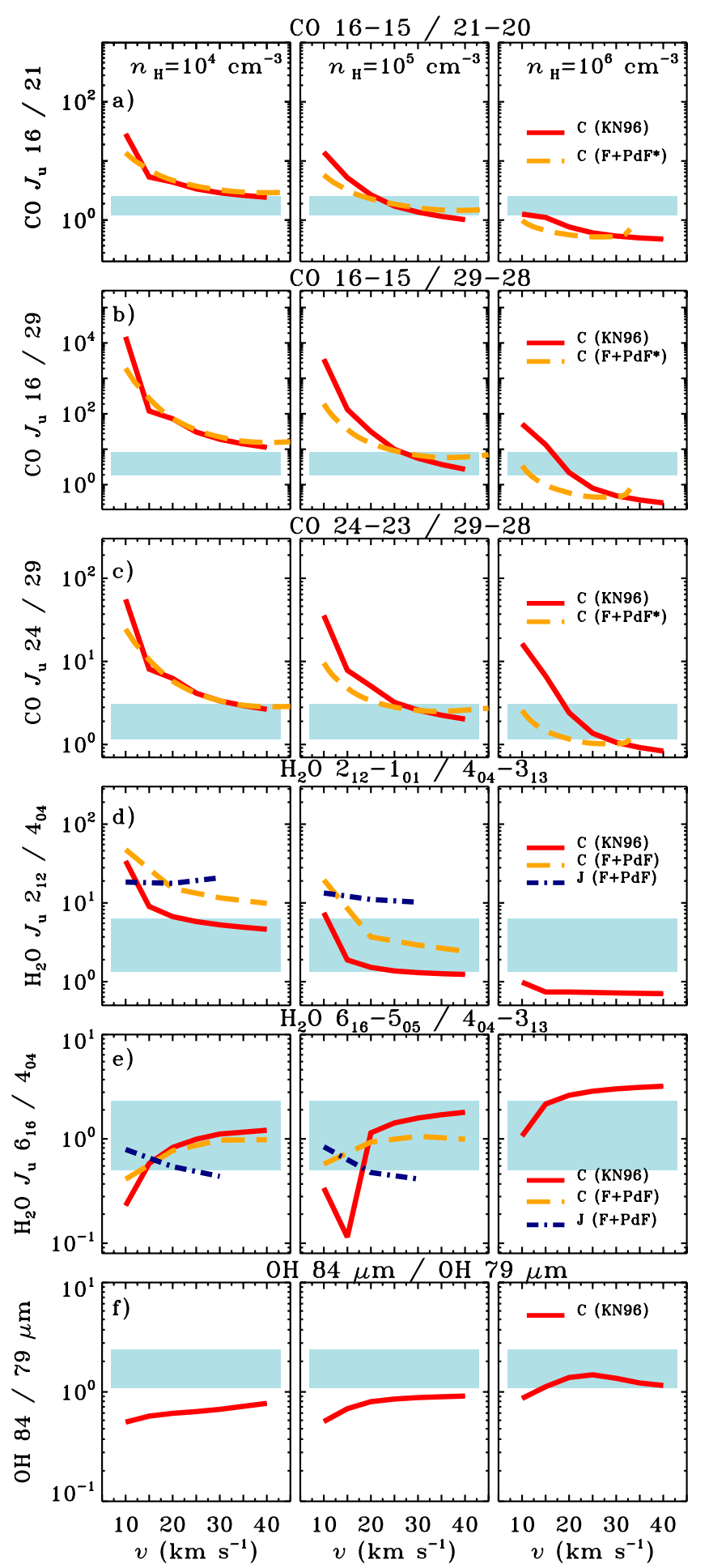

Fig. 5. Line ratios of the same species using Kaufman \& Neufeld (1996) $C$ shock models (KN96, solid line) and Flower \& Pineau des Forêts (2010) $C$ and $J$ shock models (F+PdF, dashed and dashed-dotted lines, respectively). Ratios are shown as a function of shock velocity and for pre-shock densities of $10^{4} \mathrm{~cm}^{-3}$ (left), $10^{5} \mathrm{~cm}^{-3}$ (center), and $10^{6} \mathrm{~cm}^{-3}$ (right). Observed ratios are shown as blue rectangles.

\subsubsection{CO line ratios}

Given the universal shape of the CO ladders observed toward deeply-embedded protostars (see Sect. 1 and the discussion on the origin of CO ladders in Sect. 5), three pairs of CO lines 
are compared with models: (i) CO 16-15 and 21-20 line ratio, corresponding to the "warm", $300 \mathrm{~K}$ component (panel a); (ii) $\mathrm{CO} 16-15$ and 29-28 line ratio, combining transitions located in the "warm" and "hot" (>700 K) components (panel b); (iii) CO 24-23 and 29-28, both tracing the "hot" component (panel c). All ratios are consistent with the $C$-type shock models from both KN96 and F+PdF10 for pre-shock densities above $n_{\mathrm{H}}=10^{4} \mathrm{~cm}^{-3}$. For the CO 16-15/21-20 ratio, a pre-shock density of $n_{\mathrm{H}}=10^{5} \mathrm{~cm}^{-3}$ and shock velocities of $20-30 \mathrm{~km} \mathrm{~s}^{-1}$ best fit the observations. Shock velocities above $\sim 25 \mathrm{~km} \mathrm{~s}^{-1}$ are needed to reproduce the observations of the other two ratios at the same pre-shock density. Alternatively, higher pre-shock densities with velocities below $30 \mathrm{~km} \mathrm{~s}^{-1}$ are also possible.

The KN96 $C$-shock $\mathrm{CO}$ line ratios for lower-to-higher$J$ transitions (panel b of Fig. 5) decrease with velocity, because of the increase in peak temperature that allows excitation of the higher- $J$ CO transitions. The effect is strongest at low pre-shock densities (see Sect. 4.1.1) and for the sets of transitions with the largest span in $J$ numbers. The CO 16-15/29-28 line ratio $\left(\Delta J_{\text {up }}=13\right)$ decreases by almost three orders of magnitude between shock velocities of 10 and $40 \mathrm{~km} \mathrm{~s}^{-1}$ over the range of pre-shock densities. In contrast, the CO 16-15/21-20 and CO 24-23/29-28 line ratios show drops of about one order of magnitude with increasing velocity (panel a and c of Fig. 5). These model trends explain why the observed $\mathrm{CO}$ line ratios are good diagnostics of shock velocity.

In absolute terms, the line ratios calculated for a given velocity are inversely proportional to the pre-shock density. The largest ratios obtained for $n_{\mathrm{H}}=10^{4} \mathrm{~cm}^{-3}$ result from the fact that the higher- $J$ levels are not yet populated at low shock-velocities, while the lower- $J$ transitions reach LTE at high shock-velocities and do not show an increase of flux with velocity. This effect is less prominent at higher pre-shock densities, where the higher- $J$ lines are more easily excited at low shock velocities.

The F+PdF* CO line ratios, extending the Flower \& Pineau des Forêts (2003) grid to higher- $J$ CO lines, are almost identical to the KN96 predictions for pre-shock densities $n_{\mathrm{H}}=$ $10^{4} \mathrm{~cm}^{-3}$. For higher densities, the low-velocity $C$-shock models from $\mathrm{F}+\mathrm{PdF}^{*}$ are systematically lower than the KN96 models, up to almost an order of magnitude for $10-15 \mathrm{~km} \mathrm{~s}^{-1}$ shocks at $n_{\mathrm{H}}=10^{6} \mathrm{~cm}^{-3}$. Therefore, the pre-shock density is not as well constrained solely by $\mathrm{CO}$ lines.

For densities of $10^{5} \mathrm{~cm}^{-3}$, shock velocities of $20-30 \mathrm{~km} \mathrm{~s}^{-1}$ best reproduce the ratios only using transitions from the "warm" component, while shock velocities above $25 \mathrm{~km} \mathrm{~s}^{-1}$ match the ratios using the transitions from the "hot" component. Velocities of that order are observed in CO $J=16-15$ HIFI line profiles (Kristensen et al. 2013, and in prep.), but higher- $J$ CO lines dominated by the hot component have not been obtained with sufficient velocity resolution.

\subsection{2. $\mathrm{H}_{2} \mathrm{O}$ line ratios}

Two ratios of observed $\mathrm{H}_{2} \mathrm{O}$ lines are compared with the $C$ and $J$-type shock models: (i) the ratio of the low excitation $\mathrm{H}_{2} \mathrm{O} 2_{12}-1_{01}$ and moderate excitation $4_{04}-3_{13}$ lines (panel $\mathrm{d}$ of Fig. 5) and (ii) the ratio of the highly-excited $\mathrm{H}_{2} \mathrm{O} 6_{16}-5_{05}$ and $4_{04}-3_{13}$ lines (panel e). Similar to the CO ratios, $C$-type shocks with pre-shock densities of $10^{5} \mathrm{~cm}^{-3}$ reproduce the observations well. Based on the observations of ratio (i), $\mathrm{C}$ shocks with a somewhat larger $(\mathrm{F}+\mathrm{PdF} 10)$ or smaller (KN96) pre-shock density are also possible for a broad range of shock velocities. On the other hand, no agreement with the $J$-type shocks is found for this low-excitation line ratio. Observations of ratio (ii) indicate a similar density range as ratio (i) for the KN96 models, but extend to $10^{4} \mathrm{~cm}^{-3}$ for the $\mathrm{F}+\mathrm{PdF} 10$ models, with agreement found for both $C$ - and $J$-type.

The model trends can be understood as follows. For 10-20 $\mathrm{km} \mathrm{s}^{-1}$ shocks, increasing temperature in the $C$ shock models from KN96 allows excitation of high-lying $\mathrm{H}_{2} \mathrm{O}$ lines and causes the $\mathrm{H}_{2} \mathrm{O} 2{ }_{12}-1_{01} / 4_{04}-3_{13}$ line ratio to decrease and the $\mathrm{H}_{2} \mathrm{O} 6_{16}-5_{05} / 4_{04}-3_{13}$ line ratio to increase. At higher shock velocities, the former ratio shows almost no dependence on shock velocity, while a gradual increase is seen in the ratio using two highly-excited lines in the KN96 models. At high pre-shock densities $\left(n_{\mathrm{H}}=10^{6} \mathrm{~cm}^{-3}\right)$, the upper level transitions are more easily excited and so the changes are even smaller.

The $\mathrm{H}_{2} \mathrm{O} 22_{12}-1_{01} / 4_{04}-3_{13}$ line ratios calculated using the $C$-type shock models from $\mathrm{F}+\mathrm{PdF} 10$ are a factor of a few larger than the corresponding ratios from the KN96 models (see the discussion of absolute line fluxes in Sect. 4.1.2). As a result, when compared to observations, the F+PdF10 models require pre-shock densities of at least $n_{\mathrm{H}}=10^{5} \mathrm{~cm}^{-3}$, while the KN96 models suggest densities that are lower by a factor of a few. Overall, the best fit to both the $\mathrm{CO}$ and $\mathrm{H}_{2} \mathrm{O}$ line ratios is for pre-shock densities around $10^{5} \mathrm{~cm}^{-3}$.

\subsection{3. $\mathrm{OH}$ line ratios}

Comparison of the observed $\mathrm{OH} 84$ and $79 \mu \mathrm{m}$ line ratio with the KN96 $C$-type models (panel $\mathrm{f}$ of Fig. 5) indicates an order of magnitude higher pre-shock densities, $n_{\mathrm{H}}=10^{6} \mathrm{~cm}^{-3}$, with respect to those found using the $\mathrm{CO}$ and $\mathrm{H}_{2} \mathrm{O}$ ratios. However, the KN96 models do not include any far-infrared radiation, which affects the excitation of the $\mathrm{OH}$ lines, in particular the $79 \mu \mathrm{m}$ (Wampfler et al. 2010, 2013). Additionally, part of $\mathrm{OH}$ most likely originates in a $J$-type shock, influencing our comparison (Wampfler et al. 2010; Benedettini et al. 2012; Karska et al. 2013; Kristensen et al. 2013).

Similar to the absolute fluxes of the $84 \mu \mathrm{m}$ doublet discussed in Sect. 4.1.3, not much variation in the ratio is seen with shock velocity. The ratio increases by a factor of about two between the lowest and highest pre-shock densities.

\subsection{Models and observations - line ratios of different species}

Figure 6 compares observed line ratios of various $\mathrm{H}_{2} \mathrm{O}$ and $\mathrm{CO}$ transitions with the $\mathrm{C}$ and $J$-type shock models. The line ratios of different species are sensitive both to the molecular excitation and their relative abundances.

\subsubsection{Ratios of $\mathrm{H}_{2} \mathrm{O}$ and $\mathrm{CO}$}

Comparison of observations to the KN96 and F+PdF10 models shows that the $C$-type shocks at pre-shock density $n_{\mathrm{H}}=$ $10^{5} \mathrm{~cm}^{-3}$, which best reproduces the line ratios of same species, fail to reproduce the observed line ratios of different species (Fig. 6). There are only a few cases where the observations seem to agree with the models at all. For $n_{\mathrm{H}}=10^{5} \mathrm{~cm}^{-3}$, a few $\mathrm{H}_{2} \mathrm{O} / \mathrm{CO}$ line ratios fit at low velocities $\left(<20 \mathrm{~km} \mathrm{~s}^{-1}\right.$; panels a, c and e) but this does not hold for all ratios. Moreover, such low shock velocities have been excluded in the previous section. Higher densities, $n_{\mathrm{H}}=10^{6} \mathrm{~cm}^{-3}$, are needed to reconcile the observations of the $\mathrm{H}_{2} \mathrm{O} 2_{12}-1_{01} / \mathrm{CO} 29-28$ line 

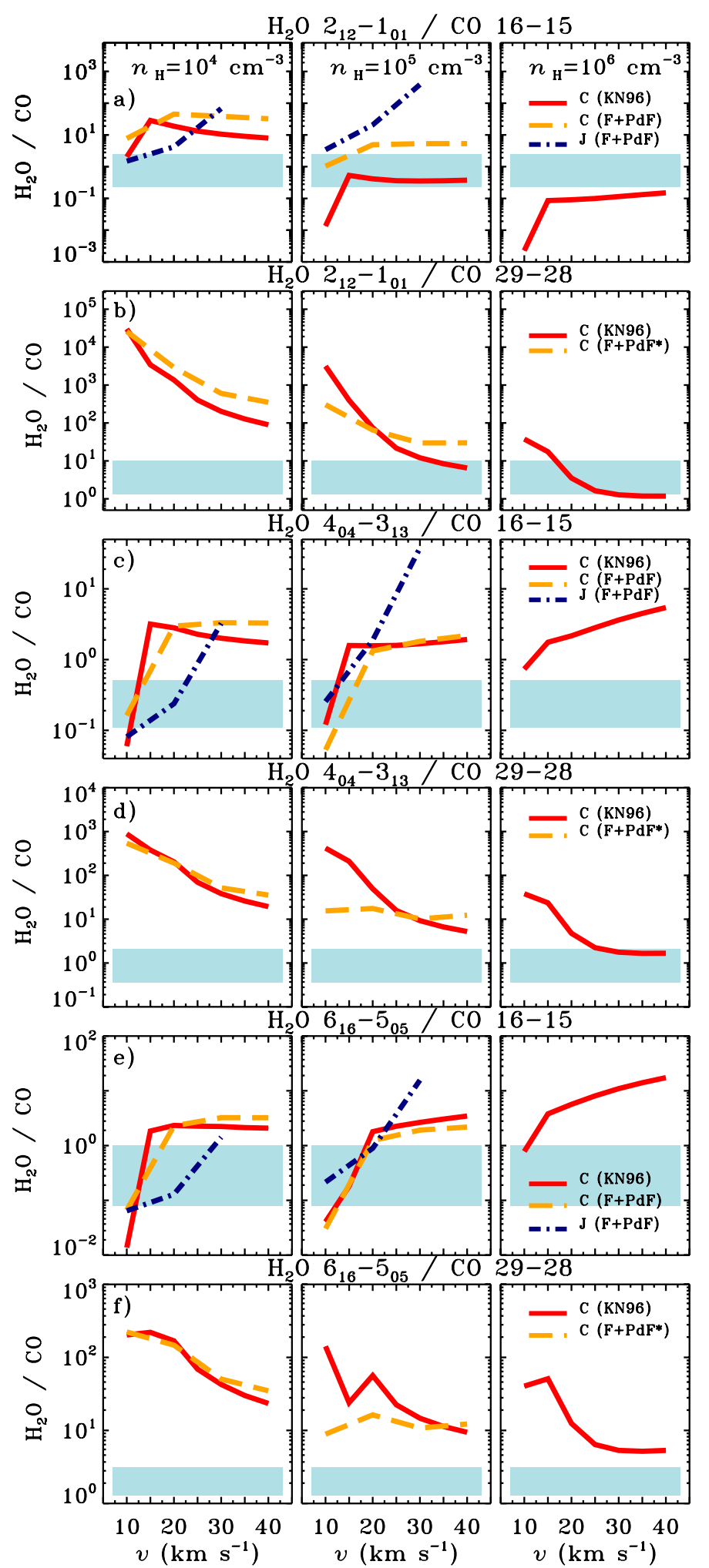

Fig. 6. $\mathrm{H}_{2} \mathrm{O}$ to $\mathrm{CO}$ line ratios using Kaufman \& Neufeld (1996) $C$ shock models (KN96, solid line) and Flower \& Pineau des Forêts (2010) $C$ and $J$ shock models ( $\mathrm{F}+\mathrm{PdF}$, dashed and dashed-dotted lines, respectively). Ratios are shown as a function of shock velocity and for pre-shock densities of $10^{4} \mathrm{~cm}^{-3}$ (left), $10^{5} \mathrm{~cm}^{-3}$ (center), and $10^{6} \mathrm{~cm}^{-3}$ (right). The $\mathrm{F}+\mathrm{PdF}$ models are available only for the pre-shock densities of $10^{4} \mathrm{~cm}^{-3}$ and $10^{5} \mathrm{~cm}^{-3}$. Observed ratios are shown as blue rectangles.

ratio. Observations of all the other ratios, using more highlyexcited $\mathrm{H}_{2} \mathrm{O}$ lines, are well below the model predictions.
The patterns seen in the panels in Fig. 6 can be understood as follows. The KN96 C type shock models show an initial rise in the $\mathrm{H}_{2} \mathrm{O} 2_{12}-1_{01}$ and $\mathrm{CO} 16-15$ line ratios from 10 to $15 \mathrm{~km} \mathrm{~s}^{-1}$ shocks (panel a), as the temperature reaches the $400 \mathrm{~K}$ and enables efficient $\mathrm{H}_{2} \mathrm{O}$ formation. Beyond this velocity, the line ratios show no variations with velocity. The decrease in this line ratio for higher densities, from 10 at $10^{4} \mathrm{~cm}^{-3}$ to 0.1 at $n_{\mathrm{H}}=10^{6} \mathrm{~cm}^{-3}$ is due to the larger increase of the column of the population in the $J_{\mathrm{u}}=16$ level with density compared to the increase in the $\mathrm{H}_{2} \mathrm{O} 2_{12}$ level (see Fig. 4 above).

Line ratios of $\mathrm{H}_{2} \mathrm{O} 22_{12}-1_{01}$ and higher- $J$ CO lines (e.g., 29-28, panel b) show more variation with velocity. A strong decrease by about an order of magnitude and up to two orders of magnitude are seen for the ratios with CO 24-23 and CO 29-28, respectively (the ratio with CO 24-23 is not shown here). These lines, as discussed in Sects. 4.1.1 and 4.2.1, are more sensitive than $\mathrm{H}_{2} \mathrm{O}$ to the increase in the maximum temperature attained in the shock that scales with shock velocities and therefore their flux is quickly rising for higher velocities (Fig. 4). The decrease is steeper for models with low pre-shock densities, since $n_{\mathrm{H}} \sim 10^{6} \mathrm{~cm}^{-3}$ allows excitation of high- $J \mathrm{CO}$ lines at lower temperatures. At this density, the $\mathrm{H}_{2} \mathrm{O} / \mathrm{CO}$ line ratios are the lowest and equal about unity.

Because of the lower CO 16-15 fluxes in the $C$-type shock models from $\mathrm{F}+\mathrm{PdF} 10$ and similar $\mathrm{H}_{2} \mathrm{O} 2_{12}-1_{01}$ fluxes (Fig. 4), the $\mathrm{H}_{2} \mathrm{O}$-to- $\mathrm{CO}$ ratios are generally larger than in the KN96 models. The exceptions are the ratios with higher- $J \mathrm{CO}$ which are more easily excited, especially at low shock velocities, in the hotter $C$-type shocks from $\mathrm{F}+\mathrm{PdF}^{*}$.

For the same reason, the increasing ratios seen in the $J$ shock models are caused by the sharp decrease in CO 16-15 flux for shock velocity $v=30 \mathrm{~km} \mathrm{~s}^{-1}$, rather than the change in the $\mathrm{H}_{2} \mathrm{O}$ lines. At such high-velocities for $J$ shocks, a significant amount of $\mathrm{CO}$ can be destroyed by reactions with hydrogen atoms (Flower \& Pineau des Forêts 2010; Suutarinen et al. 2014). Since the activation barrier for the reaction of $\mathrm{H}_{2} \mathrm{O}$ with $\mathrm{H}$ is about $10^{4} \mathrm{~K}$, the destruction of $\mathrm{H}_{2} \mathrm{O}$ does not occur until higher velocities.

Similar trends to the line ratios with $\mathrm{H}_{2} \mathrm{O} 2_{12}-1_{01}$ are seen when more highly-excited $\mathrm{H}_{2} \mathrm{O}$ lines are used (panels c-f of Fig. 6), supporting the interpretations that variations are due to differences in $\mathrm{CO}$ rather than $\mathrm{H}_{2} \mathrm{O}$ lines.

\subsubsection{Ratios of $\mathrm{CO}$ and $\mathrm{H}_{2} \mathrm{O}$ with $\mathrm{OH}$}

Figure 7 shows line ratios of $\mathrm{CO}$ or $\mathrm{H}_{2} \mathrm{O}$ with the most commonly detected $\mathrm{OH}$ doublet at $84 \mu \mathrm{m}$. The ratios are calculated for three values of pre-shock densities $\left(10^{4}, 10^{5}\right.$, and $\left.10^{6} \mathrm{~cm}^{-3}\right)$ using exclusively the KN96 models, because the F+PdF10 grid does not present $\mathrm{OH}$ fluxes.

In general, the observed $\mathrm{CO} / \mathrm{OH}$, and $\mathrm{H}_{2} \mathrm{O} / \mathrm{OH}$ ratios are similar for all sources but much lower than those predicted by the models assuming that a significant fraction of the $\mathrm{OH}$ comes from the same shock as $\mathrm{CO}$ and $\mathrm{H}_{2} \mathrm{O}$ (see Sect. 5.1). The only exception is the $\mathrm{CO} 24-23 / \mathrm{OH} 84 \mu \mathrm{m}$ ratio where models and observations agree for densities $10^{4}-10^{5} \mathrm{~cm}^{-3}$ and shock velocities below $20 \mathrm{~km} \mathrm{~s}^{-1}$. For any other set of lines discussed here, the observations do not agree with these or any other models.

As discussed in previous sections, the trends with shock velocity are determined mostly by the changes in the CO lines, rather than the $\mathrm{OH}$ itself, as seen in Fig. 4 (panel d). At shock velocities below $20 \mathrm{~km} \mathrm{~s}^{-1}$ the $\mathrm{OH}$ model flux exceeds that of $\mathrm{CO}$ owing to the abundance effect: not all $\mathrm{OH}$ has been transferred to $\mathrm{H}_{2} \mathrm{O}$ yet at low temperatures. Because of the lower 

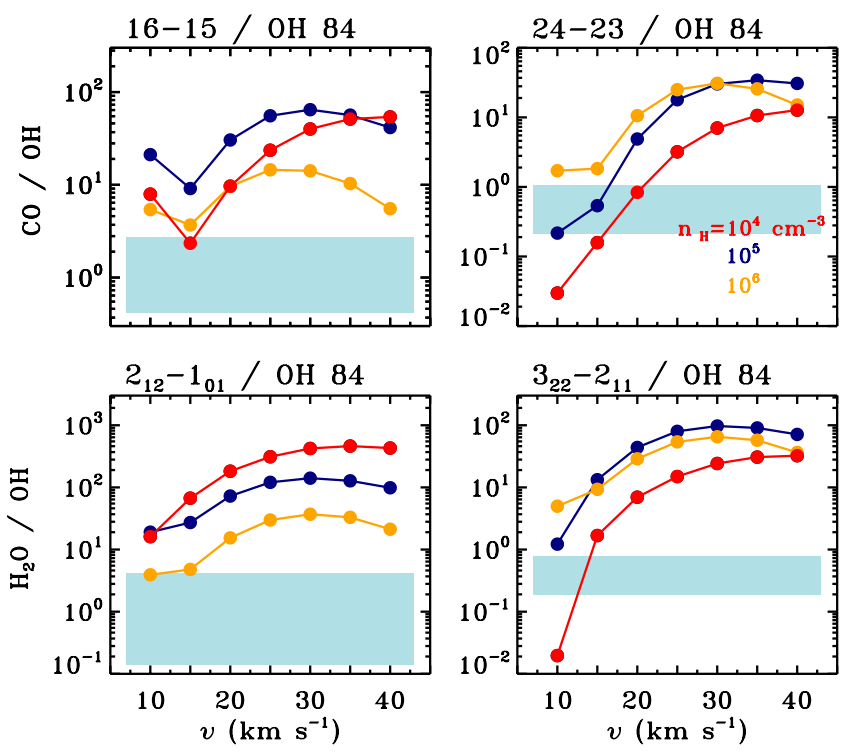

Fig. 7. $\mathrm{CO}$ to $\mathrm{OH}$ and $\mathrm{H}_{2} \mathrm{O}$ to $\mathrm{OH}$ line ratios as a function of shock velocities using KN96. The ratios are shown for pre-shock densities of $10^{4} \mathrm{~cm}^{-3}$ (red), $10^{5} \mathrm{~cm}^{-3}$ (blue), and $10^{6} \mathrm{~cm}^{-3}$ (yellow). The range of line ratios from observations is shown as filled rectangles.

critical densities of the CO lines $\left(n_{\mathrm{cr}} \sim 10^{6}-10^{7} \mathrm{~cm}^{-3}\right)$ compared with the $\mathrm{OH}$ line $\left(n_{\mathrm{cr}} \sim 10^{9} \mathrm{~cm}^{-3}\right)$, the lines for various pre-shock densities often cross and change the order in the upper panels of Fig. 7. The corresponding trends in the $\mathrm{H}_{2} \mathrm{O} / \mathrm{OH}$ line ratios are similar to those of $\mathrm{CO} / \mathrm{OH}$, except that the variations with shock velocity are smaller and the critical densities are more similar.

\section{Discussion}

\subsection{Shock parameters and physical conditions}

Spectrally resolved HIFI observations of the CO 10-9 and 16-15 line profiles (Kristensen et al. 2013 and in prep.; Yildiz et al. 2013) as well as various $\mathrm{H}_{2} \mathrm{O}$ transitions (Kristensen et al. 2012; Mottram et al. 2014) reveal at least two different kinematic shock components: non-dissociative C-type shocks in a thin layer along the cavity walls (so-called "cavity shocks") and $J$-shocks at the base of the outflow (also called "spot shocks"), both caused by interaction of the wind with the envelope. Both shocks are different from the much cooler entrained outflow gas that is observed in the low- $J$ CO line profiles (Yildiz et al. 2013).

One possible physical explanation for our observed lack of variation is that although the outflow structure depends on the mass entrainment efficiency and the amount of mass available to entrain (the envelope mass), the wind causing the shocks does not depend on these parameters. Instead the cavity shock caused by the wind impinging on the inner envelope depends on the shock velocity and the density of the inner envelope (Kristensen et al. 2013; Mottram et al. 2014). Thus, the lack of significant variation in the line ratios suggests that the shock velocities by the oblique impact of the wind are always around $20-30 \mathrm{~km} \mathrm{~s}^{-1}$.

In Sect. 4 the observed emission was compared primarily to models of $C$-type shock emission. Although $J$-type shocks play a role on small spatial scales in low-mass protostars (Kristensen et al. 2012, 2013; Mottram et al. 2014) their contribution to $\mathrm{CO}$ emission originating in levels with $J_{\text {up }} \lesssim 30$ is typically less than $\sim 50 \%$. Since higher- $J$ CO emission is only detected toward $30 \%$ of the sources, the $J$-type shock component is ignored for
CO. For the case of $\mathrm{H}_{2} \mathrm{O}$, spectrally resolved line profiles observed with HIFI reveal that the profiles do not change significantly with excitation up to $E_{\text {up }}=250 \mathrm{~K}$ (Mottram et al. 2014); $J$-type shock components typically contribute $<10 \%$ of the emission. It is unclear if the trend of line profiles not changing with excitation continues to higher upper-level energies, in particular all the way up to $E_{\text {up }}=1070 \mathrm{~K}\left(J=8_{18}-7_{07}\right.$ at $\left.63.32 \mu \mathrm{m}\right)$. $\mathrm{OH}$ and $[\mathrm{OI}]$, on the other hand, almost certainly trace dissociative $J$-type shocks (e.g., van Kempen et al. 2010; Wampfler et al. 2013) but a full analysis of their emission will be presented in a forthcoming paper. Thus, in the following the focus remains on comparing emission to models of $C$-type shocks.

Figure 8 summarizes the different line ratios as a function of pre-shock density discussed in the previous sections. General agreement is found between the observations and models when line ratios of different transitions of the same species are used (see top row for $\mathrm{H}_{2} \mathrm{O}, \mathrm{CO}$, and $\mathrm{OH}$ examples), indicating that the excitation of individual species is reproduced well by the models. The $\mathrm{H}_{2} \mathrm{O}$ line ratios are a sensitive tracer of the preshock gas density since they vary less with shock velocity than those of CO. The $C$ shock models from KN96 with pre-shock gas densities in the range of $10^{4}-10^{5} \mathrm{~cm}^{-3}$ are a best match to the observed ratios, consistent with values of $10^{5} \mathrm{~cm}^{-3}$ from the $C$ shock models of $\mathrm{F}+\mathrm{PdF} 10$. For the considered range of shock velocities, the compression factor in those shocks, defined as the ratio of the post-shock and pre-shock gas densities, varies from about 10 to 30 (Neufeld \& Dalgarno 1989; Draine \& McKee 1993; Karska et al. 2013). The resulting values of post-shock densities, traced by the observed molecules, are therefore expected to be $\geq 10^{5}-10^{6} \mathrm{~cm}^{-3}$.

The CO line ratios, on the other hand, are not only sensitive to density, but also to the shock velocities, because of their connection to the peak temperature attained in the shock. In the pre-shock density range of $\geq 10^{4}-10^{5} \mathrm{~cm}^{-3}$, indicated by the $\mathrm{H}_{2} \mathrm{O}$ line ratios, shocks with velocities above $20 \mathrm{~km} \mathrm{~s}^{-1}$ best agree with the $\mathrm{CO}$ observations. Within this range of densities, the predictions from both the KN96 and F+PdF10 $C$ shock models show a very good agreement with each other.

The ratio of two OH lines from the KN96 models compared with the observations suggest higher pre-shock densities above $10^{5} \mathrm{~cm}^{-3}$, but this ratio may be affected by infrared pumping (Wampfler et al. 2013). In addition, some $\mathrm{OH}$ emission traces (dissociative) $J$-shocks, based on its spatial connection and flux correlations to [OI] emission (Wampfler et al. 2010, 2013; Karska et al. 2013). The single spectrally-resolved OH spectrum towards Ser SMM1 (Fig. 3, Kristensen et al. 2013) suggests that the contribution of the dissociative and non-dissociative shocks is comparable. Thus, observed $\mathrm{CO} / \mathrm{OH}$ and $\mathrm{H}_{2} \mathrm{O} / \mathrm{OH}$ line ratios are only affected at the factor $\sim 2$ level and the discrepancy in the bottom row of Fig. 8 remains.

Overall, the observed $\mathrm{CO}$ and $\mathrm{H}_{2} \mathrm{O}$ line ratios are best fit with $C$-shock models with pre-shock densities of $\sim 10^{5} \mathrm{~cm}^{-3}$ and velocities $\gtrsim 20 \mathrm{~km} \mathrm{~s}^{-1}$, with higher velocities needed for the excitation of the highest- $J$ CO lines.

The shock conditions inferred here can be compared to the temperatures and densities found from single-point non-LTE excitation and radiative-transfer models, e.g., RADEX (van der Tak et al. 2007) and from non-LTE radiative transfer analysis of line intensity ratios (Kristensen et al. 2013; Mottram et al., and in prep.) toward various sources. Typically, densities $\gtrsim 10^{6} \mathrm{~cm}^{-3}$ and temperatures of $\sim 300 \mathrm{~K}$ and $\gtrsim 700 \mathrm{~K}$ are required to account for the line emission (Herczeg et al. 2012; Goicoechea et al. 2012; Santangelo et al. 2012, 2013; Vasta et al. 2012; Karska et al. 2013). Within this range of densities, the predictions from 

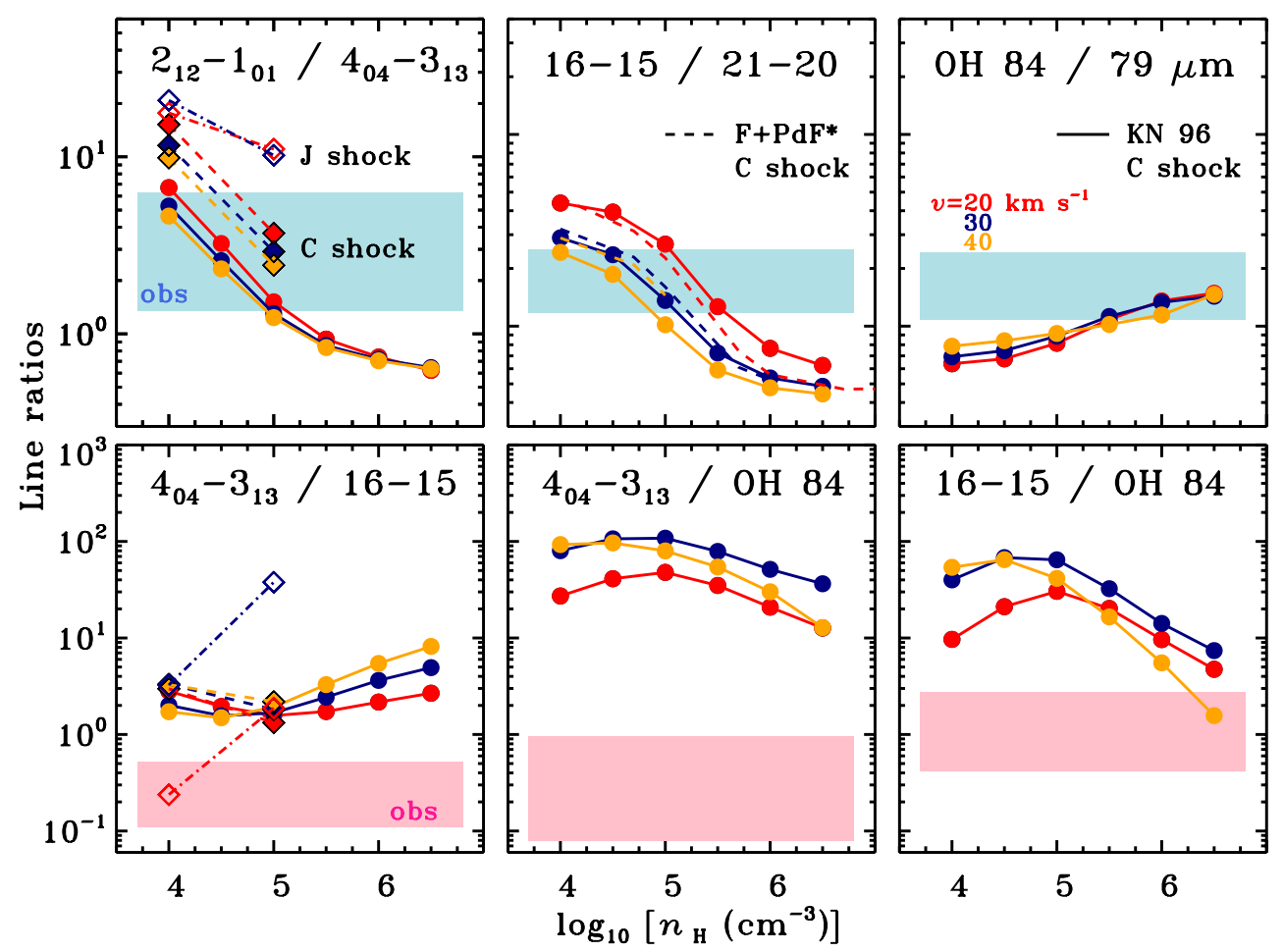

Fig. 8. Ratios of line fluxes in units of erg $\mathrm{cm}^{-2} \mathrm{~s}^{-1}$ as a function of logarithm of density of the pre-shock gas, $n_{\mathrm{H}}$. Ratios of different transitions of the same molecules are shown at the top row and line ratios comparing different species are shown at the bottom. Filled symbols and full lines show models of $C$ shocks (circles - from Kaufman \& Neufeld, diamonds - from Flower \& Pineau des Forêts), whereas the empty symbols and dash-dotted lines show models of $J$ shocks (Flower \& Pineau des Forêts 2010). Colors distinguish shock velocities: $20 \mathrm{~km} \mathrm{~s}^{-1}$ shocks are shown in red, $30 \mathrm{~km} \mathrm{~s}^{-1}$ in blue, and $40 \mathrm{~km} \mathrm{~s}^{-1}$ in orange.

both the KN96 and F+PdF10 $C$-shock models reproduce $\mathrm{CO}$ observations. However, the disconnect between predicted preshock conditions required to reproduce $\mathrm{H}_{2} \mathrm{O}$ and $\mathrm{CO}$ is puzzling (see below).

A small number of individual sources have been compared directly to shock models (Lee et al. 2013; Dionatos et al. 2013) and the conclusions are similar to what is reported here: preshock conditions of typically $10^{4}-10^{5} \mathrm{~cm}^{-3}$, and emission originating in $C$-type shocks. None of the sources analyzed previously were therefore special or atypical, rather these shock conditions appear to exist toward every embedded protostar.

At shock positions away from the protostar, dissociative or non-dissociative $J$-type shocks at the same pre-shock densities are typically invoked to explain the FIR line emission (Benedettini et al. 2012; Santangelo et al. 2012; Busquet et al. 2014). Differences between the protostar position and the distant shock positions are revealed primarily by our line ratios using the low-excitation $\mathrm{H}_{2} \mathrm{O} 2_{12}-1_{01}$ (Table 3 ) and can be ascribed to the differences in the filling factors and column densities between the immediate surrounding of the protostar and the more distant shock positions (Mottram et al. 2014).

\subsection{Abundances and need for UV radiation}

In contrast with the ratios of two $\mathrm{H}_{2} \mathrm{O}$ or $\mathrm{CO}$ lines, the ratios calculated using different species do not agree with the shock models (Fig. 8, bottom row). The ratios of $\mathrm{H}_{2} \mathrm{O}$-to-CO lines are overproduced by the $C$ shock models from both the KN96 and $\mathrm{F}+\mathrm{PdF} 10$ grids by at least an order of magnitude, irrespective of the assumed shock velocity. Although there are a few exceptions (e.g., the ratio of $\mathrm{H}_{2} \mathrm{O} 2_{12}-1_{01}$ and $\mathrm{CO} 16-15$ ), the majority of the investigated sets of $\mathrm{H}_{2} \mathrm{O}$ and $\mathrm{CO}$ lines follow the same trend.
Observations agree only with slow, $<20 \mathrm{~km} \mathrm{~s}^{-1}, J$ shock models, but as shown above, those models do not seem to reproduce the excitation properly (Fig. 5).

The discrepancy between the models and observations is even larger in the case of the $\mathrm{H}_{2} \mathrm{O}$-to- $\mathrm{OH}$ line ratios, as illustrated in Fig. 8. The two orders of magnitude disagreement with the $C$ shock models cannot be accounted by any excitation effects for any realistic shock parameters. Additional comparison to $J$ shock models is not possible because of a lack of $\mathrm{OH}$ predictions for $J$ shocks in the F+PdF10 models.

The CO-to-OH ratios are overproduced by about an order of magnitude in the $C$ shock models, similar to the $\mathrm{H}_{2} \mathrm{O}$-to-CO ratios. The agreement improves for fast $\left(v=40 \mathrm{~km} \mathrm{~s}^{-1}\right)$ shocks in high density pre-shock medium $\left(\sim 10^{6.5} \mathrm{~cm}^{-3}\right)$, but those parameters are not consistent with the line ratios from the same species.

An additional test of the disagreement between models and observations is provided by calculating the fraction of each species with regard to the sum of $\mathrm{CO}, \mathrm{H}_{2} \mathrm{O}$ and $\mathrm{OH}$ emission. For that purpose, only the strongest lines observed in our program are used. As seen in Table 4, the observed percentage (median) of $\mathrm{H}_{2} \mathrm{O}$ is about $30 \%$ and $\mathrm{OH}$ is about $25 \%$. In contrast, KN96 models predict typically 70-90\% of flux in the chosen $\mathrm{H}_{2} \mathrm{O}$ lines and only up to $2 \%$ in the $\mathrm{OH}$ lines.

The only possible way to reconcile the models with the observations, after concluding that the excitation is treated properly in the models, is to reconsider the assumed abundances. The fact that the $\mathrm{H}_{2} \mathrm{O}$-to- $\mathrm{CO}$ and $\mathrm{CO}-$ to- $\mathrm{OH}$ ratios are simultaneously overestimated suggests a problem with the abundances of $\mathrm{H}_{2} \mathrm{O}$ and $\mathrm{OH}$, rather than that of $\mathrm{CO}$. The scenario with the overestimated $\mathrm{H}_{2} \mathrm{O}$ abundances and underestimated $\mathrm{OH}$ abundances would translate into a too large $\mathrm{H}_{2} \mathrm{O}$-to- $\mathrm{OH}$ abundance 
Table 4. Fraction of $\mathrm{H}_{2} \mathrm{O}$ and $\mathrm{OH}$ emission with respect to total far-IR molecular emission.

\begin{tabular}{|c|c|c|c|c|}
\hline \multirow[t]{2}{*}{$\overline{l o g} n_{\mathrm{H}}\left(\mathrm{cm}^{-3}\right)$} & \multirow[t]{2}{*}{ Obs. (\%) } & \multicolumn{3}{|c|}{$\begin{array}{c}\text { KN96 models }(\%) \\
v\left(\mathrm{~km} \mathrm{~s}^{-1}\right):\end{array}$} \\
\hline & & 20 & 30 & 40 \\
\hline \multicolumn{5}{|c|}{$\mathrm{H}_{2} \mathrm{O} /\left(\mathrm{CO}+\mathrm{H}_{2} \mathrm{O}+\mathrm{OH}\right)$} \\
\hline 4 & 29 & 95.5 & 92.6 & 90.0 \\
\hline 4.5 & 29 & 91.6 & 86.3 & 82.8 \\
\hline 5 & 29 & 84.6 & 78.2 & 75.3 \\
\hline 5.5 & 29 & 77.3 & 71.2 & 70.4 \\
\hline 6 & 29 & 71.3 & 68.5 & 70.7 \\
\hline 6.5 & 29 & 68.3 & 69.6 & 73.3 \\
\hline \multicolumn{5}{|c|}{$\mathrm{OH} /\left(\mathrm{CO}+\mathrm{H}_{2} \mathrm{O}+\mathrm{OH}\right)$} \\
\hline 4 & 25 & 0.7 & 0.3 & 0.2 \\
\hline 4.5 & 25 & 0.7 & 0.3 & 0.3 \\
\hline 5 & 25 & 0.7 & 0.3 & 0.4 \\
\hline 5.5 & 25 & 0.9 & 0.4 & 0.6 \\
\hline 6 & 25 & 1.3 & 0.5 & 1.1 \\
\hline 6.5 & 25 & 1.9 & 0.8 & 2.4 \\
\hline
\end{tabular}

Notes. In this analysis, the following lines are used: $\mathrm{H}_{2} \mathrm{O}$ lines at $179.5 \mu \mathrm{m}, 125.4 \mu \mathrm{m}, 108.1 \mu \mathrm{m}$, and $90.0 \mu \mathrm{m}$; CO lines at $162.8 \mu \mathrm{m}$, $124.2 \mu \mathrm{m}, 108.7 \mu \mathrm{m}$, and $90.2 \mu \mathrm{m}$; $\mathrm{OH}$ lines at $79 \mu \mathrm{m}$ and $84 \mu \mathrm{m}$. Median values of the fractions calculated from 18 sources with line detections are adopted in case of observations (column Obs.).

ratio in the models. A possible and likely solution is photodissocation of $\mathrm{H}_{2} \mathrm{O}$ to $\mathrm{OH}$ and subsequently to atomic oxygen. As noted above, some $\mathrm{OH}$ also comes from the dissociative shock seen in $[\mathrm{O} \mathrm{I}]$.

A significant shortcoming of all these shock models lies in their inability to account for grain-grain interactions, which has been shown to significantly alter the structure of the shocks propagating in dense media $\left(n_{\mathrm{H}}>10^{5} \mathrm{~cm}^{-3}\right.$, Guillet et al. 2007 , 2009, 2011). These grain-grain interactions mostly consist of coagulation, vaporization, and shattering effects affecting the grains. Their inclusion in shock models necessitates a sophisticated treatment of the grains, especially following their charge and size distribution (Guillet et al. 2007). Most remarkably, such interactions eventually result in the creation of small grain fragments in large numbers, which increases the total dust grain surface area and thereby changes the coupling between the neutral and the charged fluids within the shock layer. The net effect is that the shock layer is significantly hotter and thinner (Guillet et al. 2011), which in turn affects the chemistry and emission of molecules (Guillet et al. 2009).

Unfortunately, at the moment these models are computationally expensive and are not well-suited for a grid analysis; moreover, the solutions found by Guillet et al. do not converge for preshock densities of $10^{6} \mathrm{~cm}^{-3}$ or higher. A recent study by Anderl et al. (2013) shows that it is possible to approximate these effects in a computationally efficient way, and subsequently evaluated the line intensities of $\mathrm{CO}, \mathrm{OH}$, and $\mathrm{H}_{2} \mathrm{O}$ on a small grid of models. When including grain-grain interactions, $\mathrm{CO}$ lines were found to emit significantly less than in "simpler" models, while a smaller decrease was found for $\mathrm{H}_{2} \mathrm{O}$ lines, and a small increase for $\mathrm{OH}$. These trends probably still need to be systematically investigated on larger grids of models before they can be applied to our present comparison efforts.

Regardless of the effect of grain-grain interactions, Snell et al. (2005) invoked several scenarios to reconcile high absolute $\mathrm{H}_{2} \mathrm{O}$ fluxes with the shock models for the case of supernova remnants. These include (i) the high ratio of atomic to molecular hydrogen, which drives $\mathrm{H}_{2} \mathrm{O}$ back to $\mathrm{OH}$ and $\mathrm{O}$; (ii) freeze-out of
$\mathrm{H}_{2} \mathrm{O}$ in the post-shock gas; (iii) freeze-out of $\mathrm{H}_{2} \mathrm{O}$ in the preshock gas, and (iv) photodissociation of $\mathrm{H}_{2} \mathrm{O}$ in the pre- and post-shock gas. Because of the high activation barrier of the $\mathrm{H}_{2} \mathrm{O}+\mathrm{H} \rightarrow \mathrm{OH}+\mathrm{H}_{2}$ reaction $\left(\sim 10^{4} \mathrm{~K}\right)$, the first scenario is not viable. The freeze-out in the post-shock gas (ii) is not effective in the low density regions considered in Snell et al. (2005), but can play a role in the vicinity of protostars, where densities above $\sim 10^{6} \mathrm{~cm}^{-3}$ are found (e.g., Kristensen et al. 2012, this work). However, this mechanism alone would not explain the bright $\mathrm{OH}$ and high- $J \mathrm{H}_{2} \mathrm{O}$ lines seen toward many deeply-embedded sources (e.g., Karska et al. 2013; Wampfler et al. 2013). A similar problem is related to the freeze-out in the pre-shock gas (iii), which decreases the amount of e.g., $\mathrm{O}, \mathrm{OH}$, and $\mathrm{H}_{2} \mathrm{O}$ in the gas phase for shock velocities below $15 \mathrm{~km} \mathrm{~s}^{-1}$.

Therefore, the most likely reason for the overproduction of $\mathrm{H}_{2} \mathrm{O}$ in the current generation of shock models, at the expense of $\mathrm{OH}$, is the omission of the effects of ultraviolet irradiation (scenario iv) of the shocked material. The presence of UV radiation is directly seen in Ly- $\alpha$ emission both in the outflowenvelope shocks (Curiel et al. 1995; Walter et al. 2003) and at the protostar position (Valenti et al. 2000; Yang et al. 2012). Additionally, UV radiation on scales of a few 1000 AU has been inferred from the narrow profiles of ${ }^{13} \mathrm{CO} 6-5$ observed from the ground toward a few low-mass protostars (Spaans et al. 1995; van Kempen et al. 2009; Y1ld1z et al. 2012). $\mathrm{H}_{2} \mathrm{O}$ can be photodissociated into $\mathrm{OH}$ over a broad range of far-UV wavelengths, including by Ly- $\alpha$, and this would provide an explanation for the disagreement between our observations and the models. Photodissociation of CO is less likely, given the fact that it cannot be dissociated by Ly- $\alpha$ and only by very hard UV photons with wavelengths $<1000 \AA$. The lack of CO photodissociation is consistent with weak [C I] and [C II] emission observed toward low-mass YSOs (Yildiz et al. 2012; Goicoechea et al. 2012; Karska et al. 2013). At the positions away from the protostars, on the other hand, the bow-shocks at the tip of the protostellar jets can produce significant emission in the [CI] (van Kempen et al. 2009). Therefore, it is unlikely that lower line excitation at those positions is due to the weaker UV. The differences seen in the resolved line profiles (e.g., Santangelo et al. 2012; Vasta et al. 2012; Mottram et al., in prep.) indicate that the lower column densities involved are the more likely reason for differences in the excitation.

Visser et al. (2012) proposed a scenario in which the lowerlying CO transitions observed with PACS $\left(14<J_{\mathrm{u}}<23\right)$ originate in UV-heated gas and higher- $J$ transitions $\left(J_{\mathrm{u}}>24\right)$ in shocked material. All water emission would be associated with the same shocks as responsible for the higher- $J$ CO emission with less than $1 \%$ of the water emission coming from the PDR layer. Although not modeled explicitly, the UV irradiation from the star-disk boundary impinging on the shocks naturally accounts for the lower $\mathrm{H}_{2} \mathrm{O}$ abundance and exceeds by at least two orders of magnitude the $\mathrm{H}_{2} \mathrm{O}$ destruction rate by $\mathrm{He}^{+}$and $\mathrm{H}_{3}^{+}$, assuming a normal interstellar radiation field $\left(G_{0}=1\right)$. The authors predict that while the dynamics of the hot layers where both shocks and UV irradiation play a role will be dominated by the shocks, only the UV photons penetrate farther into the envelope, where the dynamics would resemble the quiescent envelope. The lower-temperature UV-heated gas has indeed been observed to be quiescent on the spatial scales of the outflow cavity through observations of medium- $J{ }^{13} \mathrm{CO}$ lines (Yildiz et al. 2012, 2014).

Flower \& Pineau des Forêts (2013) proposed a model where all emission originates in a non-stationary shock wave, where a $J$-type shock is embedded in a $C$-type shock. Without a detailed 
modeling of individual sources based on different source parameters it is not possible to rule out any of these solutions. However, the trends reported here suggest that it is possible to find a pure shock solution, in agreement with Flower \& Pineau des Forêts (2013), as long as UV photons are incorporated to provide dissociation of $\mathrm{H}_{2} \mathrm{O}$. Complementary observations, preferably at higher angular resolution, are required to break the solution degeneracy and determine the relative role the shocks and UV photons play on the spatial scales of the thickness of the cavity wall. Models whose results depend sensitively on a single parameter such as time, are ruled out because the observed line ratios are so similar across sources.

\section{Conclusions}

We have compared the line ratios of the main molecular cooling lines detected in 22 low-mass protostars using Herschel/PACS with publicly available one-dimensional shock models. Our conclusions are the following:

- Line ratios of various species and transitions are remarkably similar for all observed sources. No correlation is found with source physical parameters.

- Line ratios observed toward the protostellar position are consistent with the values reported for the positions away from the protostar, except for some ratios involving the lowexcitation $\mathrm{H}_{2} \mathrm{O} 2_{12}-1_{01}$ line. Coupled with the larger absolute fluxes of highly-excited $\mathrm{H}_{2} \mathrm{O}$ and $\mathrm{CO}$ lines at the protostellar positions, this indicates that lines at distant off-source shock positions are less excited.

- General agreement is found between the observed line ratios of the same species $\left(\mathrm{H}_{2} \mathrm{O}, \mathrm{CO}\right.$, and $\left.\mathrm{OH}\right)$ and the $C$ shock models from Kaufman \& Neufeld (1996) and Flower \& Pineau des Forêts (2010). Ratios of $\mathrm{H}_{2} \mathrm{O}$ are particularly good tracers of the density of the ambient material and indicate pre-shock densities on the order of $\geq 10^{5} \mathrm{~cm}^{-3}$ and thus post-shock densities on the order of $10^{6} \mathrm{~cm}^{-3}$. Ratios of CO lines are more sensitive to the shock velocities and, for the derived range of pre-shock densities, indicate shock velocities above $20 \mathrm{~km} \mathrm{~s}^{-1}$.

- Ratios of CO lines located in the "warm" component of $\mathrm{CO}$ ladders (with $T_{\text {rot }} \sim 300 \mathrm{~K}$ ) are reproduced with shock velocities of $20-30 \mathrm{~km} \mathrm{~s}^{-1}$ and pre-shock densities of $10^{5} \mathrm{~cm}^{-3}$. The CO ratios using the lines from the "hot" component $\left(T_{\text {rot }} \gtrsim 700 \mathrm{~K}\right)$ are better reproduced by models with shock velocities above $25 \mathrm{~km} \mathrm{~s}^{-1}$.

- A lack of agreement is found between models and the observed line ratios of different species. The $\mathrm{H}_{2} \mathrm{O}$-to-CO, $\mathrm{H}_{2} \mathrm{O}$ to-OH, and $\mathrm{CO}-$ to-OH line ratios are all overproduced by the models by 1-2 orders of magnitude for the majority of the considered sets of transitions.

- Since the observed molecular excitation is properly reproduced in the $C$ shock models, the most likely reason for disagreement with observations is the abundances in the shock models, which are too high in case of $\mathrm{H}_{2} \mathrm{O}$ and too low in case of $\mathrm{OH}$. Invoking UV irradiation of the shocked material, together with a dissociative $J$ shock contribution to $\mathrm{OH}$ and $[\mathrm{OI}]$, would lower the $\mathrm{H}_{2} \mathrm{O}$ abundance and reconcile the models and observations.

New UV-irradiated shock models will allow us to constrain the UV field needed to reconcile the shock models with observations (M. Kaufman, priv. comm.) Those models should also account for the grain-grain processing, which affects significantly the shocks structure at densities $\sim 10^{6} \mathrm{~cm}^{-3}$ (Guillet et al. 2011). The effects of shock irradiation as a function of the distance from a protostar will help to understand the differences in the observed spectrally-resolved lines from HIFI at "on source" and distant shock-spot positions.

Acknowledgements. Herschel is an ESA space observatory with science instruments provided by European-led Principal Investigator consortia and with important participation from NASA. A.K. acknowledges support from the Polish National Science Center grant 2013/11/N/ST9/00400. Astrochemistry in Leiden is supported by the Netherlands Research School for Astronomy (NOVA), by a Royal Netherlands Academy of Arts and Sciences (KNAW) professor prize, by a Spinoza grant and grant 614.001.008 from the Netherlands Organisation for Scientific Research (NWO), and by the European Community's Seventh Framework Programme FP7/2007-2013 under grant agreement 238258 (LASSIE). N.J.E. was supported by NASA through an award issued by the Jet Propulsion Laboratory, California Institute of Technology.

\section{References}

Anderl, S., Guillet, V., Pineau des Forêts, G., \& Flower, D. R. 2013, A\&A, 556, A69

André, P., Men'shchikov, A., Bontemps, S., et al. 2010, A\&A, 518, L102

Arce, H. G., Shepherd, D., Gueth, F., et al. 2007, Protostars and Planets V, eds. B. Reipurth, D. Jewitt, \& K. Keil (University of Arizona Press), 245

Arce, H. G., Borkin, M. A., Goodman, A. A., Pineda, J. E., \& Halle, M. W. 2010, ApJ, 715, 1170

Baulch, D., Cobos, C., Cox, R., et al. 1992, J. Phys. Chem. Ref. Data, 21, 411

Benedettini, M., Busquet, G., Lefloch, B., et al. 2012, A\&A, 539, L3

Bergin, E. A., Neufeld, D. A., \& Melnick, G. J. 1998, ApJ, 499, 777

Brinch, C., \& Hogerheijde, M. R. 2010, A\&A, 523, A25

Busquet, G., Lefloch, B., Benedettini, M., et al. 2014, A\&A, 561, A120

Ceccarelli, C., Boogert, A. C. A., Tielens, A. G. G. M., et al. 2002, A\&A, 395, 863

Chieze, J.-P., Pineau des Forets, G., \& Flower, D. R. 1998, MNRAS, 295, 672

Clegg, P. E., Ade, P. A. R., Armand, C., et al. 1996, A\&A, 315, L38

Codella, C., Ceccarelli, C., Lefloch, B., et al. 2012, ApJ, 757, L9

Curiel, S., Raymond, J. C., Wolfire, M., et al. 1995, ApJ, 453, 322

Davis, C. J., Scholz, P., Lucas, P., Smith, M. D., \& Adamson, A. 2008, MNRAS, 387,954

de Graauw, T., Helmich, F. P., Phillips, T. G., et al. 2010, A\&A, 518, L6

Dionatos, O., Jørgensen, J. K., Green, J. D., et al. 2013, A\&A, 558, A88

Draine, B. T. 1980, ApJ, 241, 1021

Draine, B. T., \& McKee, C. F. 1993, ARA\&A, 31, 373

Draine, B. T., Roberge, W. G., \& Dalgarno, A. 1983, ApJ, 264, 485

Elitzur, M., \& de Jong, T. 1978, A\&A, 67, 323

Elitzur, M., \& Watson, W. D. 1978, A\&A, 70, 443

Enoch, M. L., Young, K. E., Glenn, J., et al. 2006, ApJ, 638, 293

Enoch, M. L., Evans, II, N. J., Sargent, A. I., \& Glenn, J. 2009, ApJ, 692, 973 Evans, II, N. J., Dunham, M. M., Jørgensen, J. K., et al. 2009, ApJS, 181, 321

Faure, A., Crimier, N., Ceccarelli, C., et al. 2007, A\&A, 472, 1029

Flower, D. R., \& Gusdorf, A. 2009, MNRAS, 395, 234

Flower, D. R., \& Pineau des Forêts, G. 2003, MNRAS, 343, 390

Flower, D. R., \& Pineau des Forêts, G. 2010, MNRAS, 406, 1745

Flower, D. R., \& Pineau des Forêts, G. 2012, MNRAS, 421, 2786

Flower, D. R., \& Pineau des Forêts, G. 2013, MNRAS, 436, 2143

Frank, A., Ray, T. P., Cabrit, S., et al. 2014, Protostars and Planets VI, eds. H. Beuther, R. Klessen, C. Dullemond, \& Th. Henning (University of Arizona Press), in press [arXiv: 1402.3553]

Giannini, T., Nisini, B., \& Lorenzetti, D. 2001a, ApJ, 555, 40

Giannini, T., Nisini, B., Neufeld, D., et al. 2011b, ApJ, 738, 80

Goicoechea, J. R., Cernicharo, J., Karska, A., et al. 2012, A\&A, 548, A77

Goldsmith, P. F., \& Langer, W. D. 1978, ApJ, 222, 881

Green, S., Maluendes, S., \& McLean, A. D. 1993, ApJS, 85, 181

Green, J. D., Evans, II, N. J., Jørgensen, J. K., et al. 2013, ApJ, 770, 123

Gueth, F., \& Guilloteau, S. 1999, A\&A, 343, 571

Guillet, V., Pineau Des Forêts, G., \& Jones, A. P. 2007, A\&A, 476, 263

Guillet, V., Jones, A. P., \& Pineau Des Forêts, G. 2009, A\&A, 497, 145

Guillet, V., Pineau Des Forêts, G., \& Jones, A. P. 2011, A\&A, 527, A123

Gusdorf, A., Pineau Des Forêts, G., Cabrit, S., \& Flower, D. R. 2008, A\&A, 490, 695

Gusdorf, A., Giannini, T., Flower, D. R., et al. 2011, A\&A, 532, A53

Gutermuth, R. A., Megeath, S. T., Myers, P. C., et al. 2009, ApJS, 184, 18

Gutermuth, R. A., Megeath, S. T., Myers, P. C., et al. 2010, ApJS, 189, 352

Hatchell, J., Fuller, G. A., \& Richer, J. S. 2007a, A\&A, 472, 187

Hatchell, J., Fuller, G. A., Richer, J. S., Harries, T. J., \& Ladd, E. F. 2007b, A\&A, 468, 1009 
Herczeg, G. J., Karska, A., Bruderer, S., et al. 2012, A\&A, 540, A84 Hirano, N., Ho, P. P. T., Liu, S.-Y., et al. 2010, ApJ, 717, 58

Hirota, T., Bushimata, T., Choi, Y. K., et al. 2008, PASJ, 60, 37

Hollenbach, D. 1997, in Herbig-Haro Flows and the Birth of Stars, eds. B. Reipurth, \& C. Bertout, IAU Symp., 182, 181

Hollenbach, D. J., Chernoff, D. F., \& McKee, C. F. 1989, in Infrared Spectroscopy in Astronomy, ed. E. Böhm-Vitense, ESA SP, 290, 245 Jørgensen, J. K., Harvey, P. M., Evans, II, N. J., et al. 2006, ApJ, 645, 1246 Jørgensen, J. K., Johnstone, D., Kirk, H., \& Myers, P. C. 2007, ApJ, 656, 293 Karska, A., Herczeg, G. J., van Dishoeck, E. F., et al. 2013, A\&A, 552, A141 Kaufman, M. J., \& Neufeld, D. A. 1996, ApJ, 456, 611

Kessler, M. F., Steinz, J. A., Anderegg, M. E., et al. 1996, A\&A, 315, L27

Knee, L. B. G., \& Sandell, G. 2000, A\&A, 361, 671

Kristensen, L. E., Ravkilde, T. L., Field, D., Lemaire, J. L., \& Pineau Des Forêts, G. 2007, A\&A, 469, 561

Kristensen, L. E., Visser, R., van Dishoeck, E. F., et al. 2010, A\&A, 521, L30

Kristensen, L. E., van Dishoeck, E. F., Bergin, E. A., et al. 2012, A\&A, 542, A8

Kristensen, L. E., van Dishoeck, E. F., Benz, A. O., et al. 2013, A\&A, 557, A23

Kwon, W., Looney, L. W., Crutcher, R. M., \& Kirk, J. M. 2006, ApJ, 653, 1358

Lee, J., Lee, J.-E., Lee, S., et al. 2013, ApJS, 209, 4

Lefloch, B., Cabrit, S., Busquet, G., et al. 2012a, ApJ, 757, L25

Lefloch, B., Cabrit, S., Busquet, G., et al. 2012b, ApJ, 757, L25

Lesaffre, P., Chièze, J.-P., Cabrit, S., \& Pineau des Forêts, G. 2004a, A\&A, 427, 147

Lesaffre, P., Chièze, J.-P., Cabrit, S., \& Pineau des Forêts, G. 2004b, A\&A, 427, 157

Lindberg, J. E., Jørgensen, J. K., Green, J. D., et al. 2014, A\&A, 565, A29

Looney, L. W., Mundy, L. G., \& Welch, W. J. 2000, ApJ, 529, 477

Manoj, P., Watson, D. M., Neufeld, D. A., et al. 2013, ApJ, 763, 83

Maret, S., Bergin, E. A., Neufeld, D. A., et al. 2009, ApJ, 698, 1244

Mathis, J. S., Rumpl, W., \& Nordsieck, K. H. 1977, ApJ, 217, 425

McElroy, D., Walsh, C., Markwick, A. J., et al. 2013, A\&A, 550, A36

Melnick, G. J., Stauffer, J. R., Ashby, M. L. N., et al. 2000, ApJ, 539, L77

Mottram, J., Kristensen, L., \& van Dishoeck, E. A. A. 2014, A\&A, in press [arXiv: 1409.5704]

Müller, H. S. P., Thorwirth, S., Roth, D. A., \& Winnewisser, G. 2001, A\&A, 370, L49

Müller, H. S. P., Schlöder, F., Stutzki, J., \& Winnewisser, G. 2005, J. Mol. Struct., 742,215

Neufeld, D. A. 2012, ApJ, 749, 125

Neufeld, D. A., \& Dalgarno, A. 1989, ApJ, 344, 251

Nisini, B., Benedettini, M., Giannini, T., et al. 1999, A\&A, 350, 529

Nisini, B., Benedettini, M., Giannini, T., et al. 2000, A\&A, 360, 297

Nisini, B., Giannini, T., \& Lorenzetti, D. 2002, ApJ, 574, 246

Nisini, B., Benedettini, M., Codella, C., et al. 2010a, A\&A, 518, L120
Nisini, B., Giannini, T., Neufeld, D. A., et al. 2010b, ApJ, 724, 69 Nisini, B., Santangelo, G., Antoniucci, S., et al. 2013, A\&A, 549, A16 Ott, S. 2010, in Astronomical Data Analysis Software and Systems XIX, eds. Y. Mizumoto, K.-I. Morita, \& M. Ohishi, ASP Conf. Ser., 434, 139

Pickett, H. M., Poynter, R. L., Cohen, E. A., et al. 1998, J. Quant. Spec. Radiat. Transf., 60, 883

Poglitsch, A., Waelkens, C., Geis, N., et al. 2010, A\&A, 518, L2

Rebull, L. M., Stapelfeldt, K. R., Evans, II, N. J., et al. 2007, ApJS, 171, 447

Robitaille, T. P., Whitney, B. A., Indebetouw, R., Wood, K., \& Denzmore, P. 2006, ApJS, 167, 256

Robitaille, T. P., Whitney, B. A., Indebetouw, R., \& Wood, K. 2007, ApJS, 169, 328

Sadavoy, S. I., Di Francesco, J., André, P., et al. 2014, ApJ, 787, L18

San José-García, I., Mottram, J. C., Kristensen, L. E., et al. 2013, A\&A, 553, A125

Santangelo, G., Nisini, B., Giannini, T., et al. 2012, A\&A, 538, A45

Santangelo, G., Nisini, B., Antoniucci, S., et al. 2013, A\&A, 557, A22

Santangelo, G., Nisini, B., Codella, C., et al. 2014, A\&A, 568, A125

Snell, R. L., Hollenbach, D., Howe, J. E., et al. 2005, ApJ, 620, 758

Spaans, M., Hogerheijde, M. R., Mundy, L. G., \& van Dishoeck, E. F. 1995 ApJ, 455, L167

Suutarinen, A. N., Kristensen, L. E., Mottram, J. C., Fraser, H. J., \& van Dishoeck, E. F. 2014, MNRAS, 440, 1844

Tafalla, M., Liseau, R., Nisini, B., et al. 2013, A\&A, 551, A116

Valenti, J. A., Johns-Krull, C. M., \& Linsky, J. L. 2000, ApJS, 129, 399

van Dishoeck, E. F. 2004, ARA\&A, 42, 119

van Dishoeck, E. F., Kristensen, L. E., Benz, A. O., et al. 2011, PASP, 123, 138

van Dishoeck, E. F., Herbst, E., \& Neufeld, D. A. 2013, Chem. Rev., 113, 9043

van Kempen, T. A., van Dishoeck, E. F., Güsten, R., et al. 2009, A\&A, 501, 633

van Kempen, T. A., Kristensen, L. E., Herczeg, G. J., et al. 2010, A\&A, 518, L121

Van Loo, S., Ashmore, I., Caselli, P., Falle, S. A. E. G., \& Hartquist, T. W. 2013, MNRAS, 428, 381

Vasta, M., Codella, C., Lorenzani, A., et al. 2012, A\&A, 537, A98

Velusamy, T., Langer, W. D., \& Thompson, T. 2014, ApJ, 783, 6

Visser, R., Kristensen, L. E., Bruderer, S., et al. 2012, A\&A, 537, A55

Wagner, A. F., \& Graff, M. M. 1987, ApJ, 317, 423

Walter, F. M., Herczeg, G., Brown, A., et al. 2003, AJ, 126, 3076

Wampfler, S. F., Herczeg, G. J., Bruderer, S., et al. 2010, A\&A, 521, L36

Wampfler, S. F., Bruderer, S., Karska, A., et al. 2013, A\&A, 552, A56

Yang, B., Stancil, P. C., Balakrishnan, N., \& Forrey, R. C. 2010, ApJ, 718, 1062

Yang, H., Herczeg, G. J., Linsky, J. L., et al. 2012, ApJ, 744, 121

Yildız, U. A., Kristensen, L. E., van Dishoeck, E. F., et al. 2012, A\&A, 542, A86

Yildı, U. A., Kristensen, L. E., van Dishoeck, E. F., et al. 2013, A\&A, 556, A89

Yıldız, U. A., Kristensen, L. E., van Dishoeck, E. F., et al. 2014, A\&A, submitted 


\section{Appendix A: Supplementary material}

Table A.1 provides molecular/atomic information about the lines observed in the WILL program.

Table A. 2 shows the observing log of PACS observations including observations identifications (OBSID), observation day (OD), date of observation, total integration time, and pointed coordinates (RA, Dec).

Table A.3 informs about which lines are detected toward the Perseus sources. The full list of line fluxes for all WILL sources including Perseus will be tabulated in the forthcoming paper (Karska et al., in prep.).

Table A.4 summarizes observed and modeled line ratios used in the Analysis section.

Figures A.1 and A.2 show line and continuum maps around $179.5 \mu \mathrm{m}$ for all the Perseus sources in the WILL program.

Figure A.3 show maps in the $\mathrm{H}_{2} \mathrm{O} 4_{23}-3_{12}$ line at $78.74 \mu \mathrm{m}$, OH $84.6 \mu \mathrm{m}$, and CO $29-28$ at $90.16 \mu \mathrm{m}$ for Per1, Per5, Per9, and Per20, all of which show bright line emission and centrally peaked continuum. The lines are chosen to be located close in the wavelength so that the variations in the PSF does not introduce significant changes in the emission extent.
Table A.1. Atomic and molecular data ${ }^{a}$ for lines observed in the WILL program.

\begin{tabular}{llcccc}
\hline \hline Species & Transition & $\begin{array}{c}\text { Wave. } \\
(\mu \mathrm{m})\end{array}$ & $\begin{array}{c}\text { Freq. }^{b} \\
(\mathrm{GHz})\end{array}$ & $\begin{array}{c}E_{\mathrm{u}} / k_{\mathrm{B}} \\
(\mathrm{K})\end{array}$ & $\begin{array}{c}A_{\mathrm{ul}}{ }^{c} \\
\left(\mathrm{~s}^{-1}\right)\end{array}$ \\
\hline $\mathrm{H}_{2} \mathrm{O}$ & $2_{21}-2_{12}$ & 180.488 & 1661.0 & 194.1 & $3.1(-2)$ \\
$\mathrm{H}_{2} \mathrm{O}$ & $2_{12}-1_{01}$ & 179.527 & 1669.9 & 114.4 & $5.6(-2)$ \\
$\mathrm{OH}$ & $3 / 2,1 / 2-1 / 2,1 / 2$ & 163.398 & 1834.7 & 269.8 & $2.1(-2)$ \\
$\mathrm{OH}$ & $3 / 2,1 / 2-1 / 2,1 / 2$ & 163.131 & 1837.7 & 270.1 & $2.1(-2)$ \\
$\mathrm{CO}$ & $16-15$ & 162.812 & 1841.3 & 751.7 & $4.1(-4)$ \\
{$[\mathrm{C} \mathrm{II}]$} & ${ }^{2} P_{3 / 2}-{ }^{2} P_{1 / 2}$ & 157.74 & 2060.0 & 326.6 & $1.8(-5)$ \\
$\mathrm{H}_{2} \mathrm{O}$ & $4_{04}-3_{13}$ & 125.354 & 2391.6 & 319.5 & $1.7(-1)$ \\
$\mathrm{CO}$ & $21-20$ & 124.193 & 2413.9 & 1276.1 & $8.8(-4)$ \\
$\mathrm{CO}$ & $24-23$ & 108.763 & 2756.4 & 1656.5 & $1.3(-3)$ \\
$\mathrm{H}_{2} \mathrm{O}$ & $2_{21}-1_{10}$ & 108.073 & 2774.0 & 194.1 & $2.6(-1)$ \\
$\mathrm{CO}$ & $29-28$ & 90.163 & 3325.0 & 2399.8 & $2.1(-3)$ \\
$\mathrm{H}_{2} \mathrm{O}$ & $3_{22}-211$ & 89.988 & 3331.5 & 296.8 & $3.5(-1)$ \\
$\mathrm{H}_{2} \mathrm{O}$ & $7_{16}-7_{07}$ & 84.767 & 3536.7 & 1013.2 & $2.1(-1)$ \\
$\mathrm{OH}$ & $7 / 2,3 / 2-5 / 2,3 / 2$ & 84.596 & 3543.8 & 290.5 & $4.9(-1)$ \\
$\mathrm{OH}$ & $7 / 2,3 / 2-5 / 2,3 / 2$ & 84.420 & 3551.2 & 291.2 & $2.5(-2)$ \\
$\mathrm{CO}$ & $31-30$ & 84.411 & 3551.6 & 2735.3 & $2.5(-3)$ \\
$\mathrm{H}_{2} \mathrm{O}$ & $6_{16}-5_{05}$ & 82.032 & 3654.6 & 643.5 & $7.5(-1)$ \\
$\mathrm{CO}$ & $32-31$ & 81.806 & 3664.7 & 2911.2 & $2.7(-3)$ \\
$\mathrm{CO}$ & $33-32$ & 79.360 & 3777.6 & 3092.5 & $3.0(-3)$ \\
$\mathrm{OH}$ & $1 / 2,1 / 2-3 / 2,3 / 2$ & 79.182 & 3786.1 & 181.7 & $2.9(-2)$ \\
$\mathrm{OH}$ & $1 / 2,1 / 2-3 / 2,3 / 2$ & 79.116 & 3789.3 & 181.9 & $5.8(-3)$ \\
$\mathrm{H}_{2} \mathrm{O}$ & $6_{15}-5_{24}$ & 78.928 & 3798.3 & 781.1 & $4.6(-1)$ \\
$\mathrm{H}_{2} \mathrm{O}$ & $4_{23}-3_{12}$ & 78.742 & 3807.3 & 432.2 & $4.9(-1)$ \\
$\mathrm{H}_{2} \mathrm{O}$ & $8_{18}-7_{07}$ & 63.324 & 4734.3 & 1070.7 & 1.8 \\
{$[\mathrm{OI}]$} & ${ }^{3} P_{1}-{ }^{3} P_{2}$ & 63.184 & 4744.8 & 227.7 & $8.9(-5)$ \\
\hline & & & & &
\end{tabular}

Notes. ${ }^{(a)}$ Compiled using the CDMS (Müller et al. 2001, 2005) and JPL (Pickett et al. 1998) databases. ${ }^{(b)}$ Frequencies are rest frequencies. (c) $A(B) \equiv A \times 10^{B}$. 
Table A.2. Log of PACS observations.

\begin{tabular}{|c|c|c|c|c|c|c|}
\hline Source & OBSID & OD & Date & $\begin{array}{l}\text { Total time } \\
\text { (s) }\end{array}$ & $\begin{array}{c}\mathrm{RA} \\
(\mathrm{h} \mathrm{m} \mathrm{s})\end{array}$ & $\begin{array}{c}\text { Dec } \\
\left({ }^{\prime}, \prime \prime\right)\end{array}$ \\
\hline \multirow[t]{2}{*}{ Per01 } & 1342263508 & 1370 & $2013-02-12$ & 851 & 32522.32 & +304513.9 \\
\hline & 1342263509 & 1370 & 2013-02-12 & 1986 & 32522.32 & +304513.9 \\
\hline \multirow[t]{2}{*}{ Per02 } & 1342263506 & 1370 & 2013-02-12 & 851 & 32536.49 & +304522.2 \\
\hline & 1342263507 & 1370 & 2013-02-12 & 1986 & 32536.49 & +304522.2 \\
\hline \multirow[t]{2}{*}{ Per03 } & 1342263510 & 1370 & $2013-02-12$ & 851 & 32539.12 & +304358.2 \\
\hline & 1342263511 & 1370 & 2013-02-12 & 1986 & 32539.12 & +304358.2 \\
\hline \multirow[t]{2}{*}{ Per04 } & 1342264250 & 1383 & $2013-02-25$ & 851 & 32637.47 & +301528.1 \\
\hline & 1342264251 & 1383 & $2013-02-25$ & 1986 & 32637.47 & +301528.1 \\
\hline \multirow{2}{*}{ Per05 } & 1342264248 & 1383 & $2013-02-25$ & 851 & 32837.09 & +311330.8 \\
\hline & 1342264249 & 1383 & $2013-02-25$ & 1986 & 32837.09 & +311330.8 \\
\hline \multirow[t]{2}{*}{ Per06 } & 1342264247 & 1383 & $2013-02-25$ & 1986 & 32857.36 & +311415.9 \\
\hline & 1342264246 & 1383 & 2013-02-25 & 851 & 32857.36 & +31 1415.9 \\
\hline \multirow{2}{*}{ Per07 } & 1342264244 & 1383 & $2013-02-25$ & 1986 & 32900.55 & +311200.8 \\
\hline & 1342264245 & 1383 & $2013-02-25$ & 851 & 32900.55 & +311200.8 \\
\hline \multirow[t]{2}{*}{ Per08 } & 1342264242 & 1383 & $2013-02-25$ & 1986 & 32901.56 & +312020.6 \\
\hline & 1342264243 & 1383 & $2013-02-25$ & 851 & 32901.56 & +312020.6 \\
\hline \multirow[t]{2}{*}{ Per09 } & 1342267611 & 1401 & 2013-03-15 & 1986 & 32907.78 & +312157.3 \\
\hline & 1342267612 & 1401 & 2013-03-15 & 851 & 32907.78 & +312157.3 \\
\hline \multirow[t]{2}{*}{ Per10 } & 1342267615 & 1401 & 2013-03-15 & 1986 & 32910.68 & +311820.6 \\
\hline & 1342267616 & 1401 & 2013-03-15 & 851 & 32910.68 & +311820.6 \\
\hline \multirow[t]{2}{*}{ Per11 } & 1342267607 & 1401 & 2013-03-15 & 1986 & 32912.06 & +311301.7 \\
\hline & 1342267608 & 1401 & 2013-03-15 & 851 & 32912.06 & +311301.7 \\
\hline \multirow[t]{2}{*}{ Per12 } & 1342267609 & 1401 & 2013-03-15 & 1986 & 32913.54 & +311358.2 \\
\hline & 1342267610 & 1401 & 2013-03-15 & 851 & 32913.54 & +311358.2 \\
\hline \multirow[t]{2}{*}{ Per13 } & 1342267613 & 1401 & 2013-03-15 & 1986 & 32951.82 & +313906.0 \\
\hline & 1342267614 & 1401 & 2013-03-15 & 851 & 32951.82 & +313906.0 \\
\hline \multirow[t]{2}{*}{ Per14 } & 1342263512 & 1370 & 2013-02-12 & 1986 & 33015.14 & +302349.4 \\
\hline & 1342263513 & 1370 & 2013-02-12 & 851 & 33015.14 & +302349.4 \\
\hline \multirow[t]{2}{*}{ Per15 } & 1342263514 & 1370 & 2013-02-12 & 1986 & 33120.98 & +304530.1 \\
\hline & 1342263515 & 1370 & 2013-02-12 & 851 & 33120.98 & +304530.1 \\
\hline \multirow[t]{2}{*}{ Per16 } & 1342265447 & 1374 & 2013-02-16 & 1986 & 33217.96 & +304947.5 \\
\hline & 1342265448 & 1374 & 2013-02-16 & 851 & 33217.96 & +304947.5 \\
\hline \multirow[t]{2}{*}{ Per17 } & 1342263486 & 1369 & 2013-02-11 & 1986 & 33314.38 & +310710.9 \\
\hline & 1342263487 & 1369 & 2013-02-11 & 851 & 33314.38 & +310710.9 \\
\hline \multirow[t]{2}{*}{ Per18 } & 1342265449 & 1374 & $2013-02-16$ & 1986 & 33316.44 & +310652.5 \\
\hline & 1342265450 & 1374 & 2013-02-16 & 851 & 33316.44 & +310652.5 \\
\hline \multirow[t]{2}{*}{ Per19 } & 1342265451 & 1374 & 2013-02-16 & 1986 & 33327.29 & +310710.2 \\
\hline & 1342265452 & 1374 & 2013-02-16 & 851 & 33327.29 & +310710.2 \\
\hline \multirow[t]{2}{*}{ Per20 } & 1342265453 & 1374 & 2013-02-16 & 1986 & 34356.52 & +320052.8 \\
\hline & 1342265454 & 1374 & 2013-02-16 & 851 & 34356.52 & +320052.8 \\
\hline \multirow[t]{2}{*}{ Per21 } & 1342265455 & 1374 & 2013-02-16 & 1986 & 34356.84 & +320304.7 \\
\hline & 1342265456 & 1374 & $2013-02-16$ & 851 & 34356.84 & +320304.7 \\
\hline \multirow[t]{2}{*}{ Per22 } & 1342265701 & 1381 & $2013-02-23$ & 1986 & 34443.96 & +320136.2 \\
\hline & 1342265702 & 1381 & $2013-02-23$ & 851 & 34443.96 & +320136.2 \\
\hline
\end{tabular}


Table A.3. Line detections toward our Perseus sources.

\begin{tabular}{|c|c|c|c|c|c|c|c|c|c|c|c|c|c|c|c|c|c|c|c|c|c|c|c|}
\hline Wave $(\mu \mathrm{m})$ & Species & Per 1 & 2 & 3 & 4 & 5 & 6 & 7 & 8 & 9 & 10 & 11 & 12 & 13 & 14 & 15 & 16 & 17 & 18 & 19 & 20 & 21 & 22 \\
\hline 63.184 & [O I] & $\checkmark$ & $\checkmark$ & $\checkmark$ & $\checkmark$ & $\checkmark$ & $\checkmark$ & $\checkmark$ & $\checkmark$ & $\checkmark$ & $\checkmark$ & $\checkmark$ & $\checkmark$ & - & $\checkmark$ & $\checkmark$ & $\checkmark$ & $\checkmark$ & $\checkmark$ & $\checkmark$ & $\checkmark$ & $\checkmark$ & $\checkmark$ \\
\hline 63.324 & $\mathrm{H}_{2} \mathrm{O}$ & $\checkmark$ & $\checkmark$ & $\checkmark$ & - & - & $\checkmark$ & - & $\checkmark$ & $\checkmark$ & - & $\checkmark$ & - & - & $\checkmark$ & - & - & - & - & - & - & - & - \\
\hline 63.458 & $\mathrm{H}_{2} \mathrm{O}$ & - & - & $\checkmark$ & - & - & - & - & $\checkmark$ & $\checkmark$ & - & $\checkmark$ & - & - & - & _- & - & - & - & - & - & - & - \\
\hline 78.742 & $\mathrm{H}_{2} \mathrm{O}$ & $\checkmark$ & $\checkmark$ & $\checkmark$ & - & $\checkmark$ & $\checkmark$ & - & $\checkmark$ & $\checkmark$ & - & $\checkmark$ & - & - & $\checkmark$ & - & - & - & - & - & $\checkmark$ & $\checkmark$ & $\checkmark$ \\
\hline 78.928 & $\mathrm{H}_{2} \mathrm{O}$ & $\checkmark$ & $\checkmark$ & $\checkmark$ & - & $\checkmark$ & - & - & - & $\checkmark$ & - & $\checkmark$ & - & - & - & - & - & - & - & - & - & - & - \\
\hline 79.120 & $\mathrm{OH}$ & $\checkmark$ & $\checkmark$ & $\checkmark$ & - & $\checkmark$ & $\checkmark$ & - & $\checkmark$ & $\checkmark$ & $\checkmark$ & $\checkmark$ & - & - & $\checkmark$ & - & - & - & - & $\checkmark$ & $\checkmark$ & $\checkmark$ & $\checkmark$ \\
\hline 79.180 & $\mathrm{OH}$ & $\checkmark$ & $\checkmark$ & $\checkmark$ & - & $\checkmark$ & $\checkmark$ & - & $\checkmark$ & $\checkmark$ & $\checkmark$ & $\checkmark$ & - & - & $\checkmark$ & - & - & - & - & $\checkmark$ & $\checkmark$ & $\checkmark$ & $\checkmark$ \\
\hline 79.360 & $\mathrm{CO}$ & $\checkmark$ & $\checkmark$ & $\checkmark$ & - & $\checkmark$ & - & - & - & $\checkmark$ & - & $\checkmark$ & - & - & $\checkmark$ & - & - & - & - & - & - & - & - \\
\hline 81.806 & $\mathrm{CO}$ & $\checkmark$ & $\checkmark$ & $\checkmark$ & - & - & $\checkmark$ & - & $\checkmark$ & $\checkmark$ & - & $\checkmark$ & - & - & $\checkmark$ & - & - & - & - & - & - & - & - \\
\hline 82.032 & $\mathrm{H}_{2} \mathrm{O}$ & $\checkmark$ & $\checkmark$ & $\checkmark$ & - & $\checkmark$ & $\checkmark$ & - & $\checkmark$ & $\checkmark$ & - & $\checkmark$ & - & - & $\checkmark$ & $\checkmark$ & - & - & - & $\checkmark$ & - & $\checkmark$ & $\checkmark$ \\
\hline 84.411 & $\mathrm{OH}+\mathrm{CO}$ & $\checkmark$ & $\checkmark$ & $\checkmark$ & - & $\checkmark$ & $\checkmark$ & - & $\checkmark$ & $\checkmark$ & $\checkmark$ & $\checkmark$ & - & - & $\checkmark$ & $\checkmark$ & - & - & - & $\checkmark$ & $\checkmark$ & $\checkmark$ & $\checkmark$ \\
\hline 84.600 & $\mathrm{OH}$ & $\checkmark$ & $\checkmark$ & $\checkmark$ & - & $\checkmark$ & $\checkmark$ & - & $\checkmark$ & $\checkmark$ & $\checkmark$ & $\checkmark$ & - & - & $\checkmark$ & $\checkmark$ & - & - & - & $\checkmark$ & $\checkmark$ & $\checkmark$ & $\checkmark$ \\
\hline 84.767 & $\mathrm{H}_{2} \mathrm{O}$ & - & - & $\checkmark$ & - & - & - & - & - & - & - & - & - & - & - & - & - & - & - & - & - & - & - \\
\hline 89.988 & $\mathrm{H}_{2} \mathrm{O}$ & $\checkmark$ & $\checkmark$ & $\checkmark$ & - & $\checkmark$ & - & - & $\checkmark$ & $\checkmark$ & - & $\checkmark$ & - & - & $\checkmark$ & - & - & - & - & - & - & $\checkmark$ & - \\
\hline 90.163 & $\mathrm{CO}$ & $\checkmark$ & $\checkmark$ & $\checkmark$ & - & $\checkmark$ & - & - & $\checkmark$ & $\checkmark$ & $\checkmark$ & $\checkmark$ & - & - & $\checkmark$ & - & - & - & - & - & - & $\checkmark$ & - \\
\hline 108.073 & $\mathrm{H}_{2} \mathrm{O}$ & $\checkmark$ & $\checkmark$ & $\checkmark$ & - & $\checkmark$ & $\checkmark$ & - & $\checkmark$ & $\checkmark$ & $\checkmark$ & $\checkmark$ & - & $\checkmark$ & $\checkmark$ & $\checkmark$ & - & - & $\checkmark$ & $\checkmark$ & $\checkmark$ & $\checkmark$ & $\checkmark$ \\
\hline 108.763 & $\mathrm{CO}$ & $\checkmark$ & $\checkmark$ & $\checkmark$ & - & $\checkmark$ & $\checkmark$ & - & $\checkmark$ & $\checkmark$ & $\checkmark$ & $\checkmark$ & - & - & $\checkmark$ & $\checkmark$ & - & - & $\checkmark$ & $\checkmark$ & $\checkmark$ & $\checkmark$ & $\checkmark$ \\
\hline 124.193 & $\mathrm{CO}$ & $\checkmark$ & $\checkmark$ & $\checkmark$ & - & $\checkmark$ & $\checkmark$ & - & $\checkmark$ & $\checkmark$ & $\checkmark$ & $\checkmark$ & - & - & $\checkmark$ & $\checkmark$ & $\checkmark$ & - & $\checkmark$ & $\checkmark$ & $\checkmark$ & $\checkmark$ & $\checkmark$ \\
\hline 125.354 & $\mathrm{H}_{2} \mathrm{O}$ & $\checkmark$ & $\checkmark$ & $\checkmark$ & - & $\checkmark$ & $\checkmark$ & - & $\checkmark$ & $\checkmark$ & $\checkmark$ & $\checkmark$ & - & $\checkmark$ & $\checkmark$ & $\checkmark$ & - & - & - & $\checkmark$ & $\checkmark$ & $\checkmark$ & $\checkmark$ \\
\hline 162.812 & $\mathrm{CO}$ & $\checkmark$ & $\checkmark$ & $\checkmark$ & - & $\checkmark$ & $\checkmark$ & - & $\checkmark$ & $\checkmark$ & $\checkmark$ & $\checkmark$ & - & - & $\checkmark$ & $\checkmark$ & $\checkmark$ & - & $\checkmark$ & $\checkmark$ & $\checkmark$ & $\checkmark$ & $\checkmark$ \\
\hline 163.120 & $\mathrm{OH}$ & $\checkmark$ & $\checkmark$ & $\checkmark$ & - & - & $\checkmark$ & - & $\checkmark$ & $\checkmark$ & $\checkmark$ & $\checkmark$ & - & - & $\checkmark$ & - & - & - & $\checkmark$ & $\checkmark$ & $\checkmark$ & - & $\checkmark$ \\
\hline 163.400 & $\mathrm{OH}$ & $\checkmark$ & $\checkmark$ & $\checkmark$ & - & - & $\checkmark$ & - & $\checkmark$ & $\checkmark$ & $\checkmark$ & $\checkmark$ & - & - & $\checkmark$ & $\checkmark$ & - & - & $\checkmark$ & $\checkmark$ & $\checkmark$ & $\checkmark$ & - \\
\hline 179.527 & $\mathrm{H}_{2} \mathrm{O}$ & $\checkmark$ & $\checkmark$ & $\checkmark$ & - & $\checkmark$ & $\checkmark$ & - & $\checkmark$ & $\checkmark$ & $\checkmark$ & $\checkmark$ & - & - & $\checkmark$ & $\checkmark$ & - & - & $\checkmark$ & $\checkmark$ & $\checkmark$ & $\checkmark$ & $\checkmark$ \\
\hline 180.488 & $\mathrm{H}_{2} \mathrm{O}$ & $\checkmark$ & $\checkmark$ & $\checkmark$ & - & $\checkmark$ & - & - & - & $\checkmark$ & - & $\checkmark$ & - & - & $\checkmark$ & - & - & - & $\checkmark$ & $\checkmark$ & $\checkmark$ & $\checkmark$ & $\checkmark$ \\
\hline
\end{tabular}

Table A.4. Observed and model line ratios based on Kaufman \& Neufeld (1996).

\begin{tabular}{|c|c|c|c|c|c|c|c|c|c|c|c|c|c|c|c|}
\hline \multirow[t]{3}{*}{ Line 1} & \multirow[t]{3}{*}{ Line 2} & \multirow{2}{*}{\multicolumn{5}{|c|}{$\begin{array}{l}\text { Observed line ratios } \\
\text { Line 1/Line } 2\end{array}$}} & \multicolumn{9}{|c|}{ Models $-C$ shock (KN96) } \\
\hline & & & & & & & & $\mathrm{g} n_{\mathrm{H}}=$ & & & $n_{\mathrm{H}}=$ & & & $n_{\mathrm{H}}$ & \\
\hline & & $\mathrm{N}$ & Min & $\operatorname{Max}$ & Mean & Std & $v=20$ & 30 & 40 & 20 & 30 & 40 & 20 & 30 & 40 \\
\hline CO $16-15$ & $21-20$ & 17 & 1.2 & 2.5 & 1.7 & 0.4 & 4.4 & 2.9 & 2.4 & 2.7 & 1.4 & 1.0 & 0.8 & 0.5 & 0.5 \\
\hline CO $16-15$ & $24-23$ & 16 & 1.2 & 4.6 & 2.3 & 0.9 & 11.6 & 5.7 & 4.2 & 6.2 & 2.1 & 1.3 & 0.9 & 0.5 & 0.4 \\
\hline CO $16-15$ & $29-28$ & 10 & 1.9 & 8.3 & 3.8 & 1.8 & 72.2 & 31.4 & 2.2 & 19.1 & 5.5 & 0.5 & 11.3 & 2.7 & 0.3 \\
\hline CO 21-20 & $29-28$ & 10 & 1.4 & 4.1 & 2.2 & 0.8 & 16.5 & 11.7 & 2.9 & 6.6 & 4.1 & 0.9 & 4.6 & 2.7 & 0.6 \\
\hline $\mathrm{H}_{2} \mathrm{O} 2_{12}-1_{01}$ & $4_{04}-3_{13}$ & 15 & 1.3 & 6.3 & 3.0 & 1.6 & 6.7 & 5.3 & 4.6 & 1.5 & 1.3 & 1.2 & 0.7 & 0.7 & 0.7 \\
\hline $\mathrm{H}_{2} \mathrm{O} 2_{12}-1_{01}$ & $6_{16}-5_{05}$ & 13 & 0.7 & 5.9 & 2.4 & 1.8 & 8.1 & 4.7 & 3.8 & 1.3 & 0.8 & 0.7 & 0.3 & 0.2 & 0.2 \\
\hline $\mathrm{H}_{2} \mathrm{O} 2_{21}-1_{10}$ & $4_{04}-3_{13}$ & 16 & 1.4 & 5.5 & 2.8 & 1.1 & 4.2 & 3.6 & 3.3 & 2.3 & 2.0 & 1.8 & 1.5 & 1.3 & 1.3 \\
\hline $\mathrm{H}_{2} \mathrm{O} 2_{21}-1_{10}$ & $6_{16}-5_{05}$ & 13 & 0.9 & 5.5 & 2.1 & 1.3 & 5.0 & 3.2 & 2.7 & 2.0 & 1.2 & 1.0 & 0.6 & 0.4 & 0.4 \\
\hline $\mathrm{OH} 84$ & OH 79 & 14 & 1.1 & 2.4 & 1.7 & 0.3 & 0.6 & 0.7 & 0.8 & 0.8 & 0.9 & 0.9 & 1.4 & 1.3 & 1.1 \\
\hline $\mathrm{H}_{2} \mathrm{O} 2_{12}-1_{01}$ & CO 16-15 & 16 & 0.2 & 2.4 & $\overline{0.9}$ & 0.8 & 18.8 & 10.6 & 8.0 & 0.4 & 0.4 & 0.4 & 0.1 & 0.1 & 0.1 \\
\hline $\mathrm{H}_{2} \mathrm{O}_{22}-1_{10}$ & CO $24-23$ & 16 & 0.6 & 3.8 & 1.7 & 1.0 & 135.7 & 41.1 & 24.2 & 22.5 & 7.0 & 4.8 & 3.0 & 2.2 & 2.5 \\
\hline $\mathrm{H}_{2} \mathrm{O}_{22}-2_{11}$ & CO 29-28 & 9 & 0.7 & 2.1 & 1.2 & 0.4 & 51.9 & 11.6 & 6.7 & 45.3 & 8.3 & 4.7 & 6.6 & 2.2 & 2.0 \\
\hline $\mathrm{H}_{2} \mathrm{O} 4_{04}-3_{13}$ & CO $16-15$ & 15 & 0.1 & 0.5 & 0.3 & 0.1 & 2.8 & 2.0 & 1.7 & 1.6 & 1.7 & 1.9 & 2.2 & 3.6 & 5.4 \\
\hline $\mathrm{H}_{2} \mathrm{O} 4_{04}-3_{13}$ & CO 21-20 & 15 & 0.2 & 0.9 & 0.5 & 0.2 & 12.3 & 5.8 & 4.2 & 4.2 & 2.3 & 2.0 & 1.7 & 2.0 & 2.6 \\
\hline $\mathrm{H}_{2} \mathrm{O} 6_{16}-5_{05}$ & CO $16-15$ & 13 & 0.1 & 1.0 & 0.5 & 0.3 & 2.3 & 2.2 & 2.1 & 0.05 & 0.07 & 0.1 & 0.01 & 0.02 & 0.04 \\
\hline $\mathrm{H}_{2} \mathrm{O} 6_{16}-5_{05}$ & CO 21-20 & 13 & 0.2 & 1.4 & 0.7 & 0.4 & 10.2 & 6.5 & 5.1 & 0.1 & 0.1 & 0.1 & 0.01 & 0.01 & 0.02 \\
\hline $\mathrm{H}_{2} \mathrm{O}_{616}-5_{05}$ & CO 24-23 & 13 & 0.4 & 1.6 & 0.9 & 0.5 & 27.0 & 12.7 & 8.8 & 11.2 & 5.7 & 4.7 & 5.2 & 5.0 & 6.4 \\
\hline $\mathrm{H}_{2} \mathrm{O}_{616}-5_{05}$ & CO 32-31 & 8 & 1.2 & 4.6 & 3.0 & 1.1 & 576.3 & 91.6 & 43.3 & 165.4 & 28.1 & 15.6 & 28.6 & 6.6 & 5.5 \\
\hline $\mathrm{H}_{2} \mathrm{O} 2_{12}-1_{01}$ & $\mathrm{OH} 84$ & 15 & 0.1 & 4.2 & 1.2 & 1.3 & 182.2 & 422.8 & 429.4 & 72.5 & 140.7 & 98.6 & 15.4 & 37.0 & 21.2 \\
\hline $\mathrm{H}_{2} \mathrm{O}_{22}-2_{11}$ & $\mathrm{OH} 84$ & 9 & 0.2 & 0.8 & 0.4 & 0.2 & 7.0 & 24.2 & 32.4 & 43.9 & 97.3 & 71.2 & 28.8 & 65.3 & 36.5 \\
\hline CO 16-15 & $\mathrm{OH} 84$ & 15 & 0.4 & 2.8 & 1.1 & 0.7 & 9.7 & 40.0 & 54.0 & 30.4 & 64.6 & 41.5 & 9.6 & 14.2 & 5.5 \\
\hline CO $24-23$ & $\mathrm{OH} 84$ & 15 & 0.2 & 1.1 & 0.5 & 0.3 & 0.8 & 7.0 & 12.7 & 4.9 & 30.3 & 30.8 & 10.6 & 30.8 & 15.1 \\
\hline
\end{tabular}

Notes. Number of YSOs with detections of the two lines in the ratio (N), minimum (Min) and maximum (Max) value of the ratio, mean ratio value (Mean) and standard deviation (Std) is given for the observed line ratios. Model line ratios are calculated for three values of pre-shock densities, $n_{\mathrm{H}}=10^{4}, 10^{5}$, and $10^{6} \mathrm{~cm}^{-3}$, and three values of shock velocities, $v=20,30$, and $40 \mathrm{~km} \mathrm{~s}^{-1}$. See Table A.1 for the full identifiers of the lines. 
A. Karska et al.: Water deficit in low-mass YSOs in Perseus
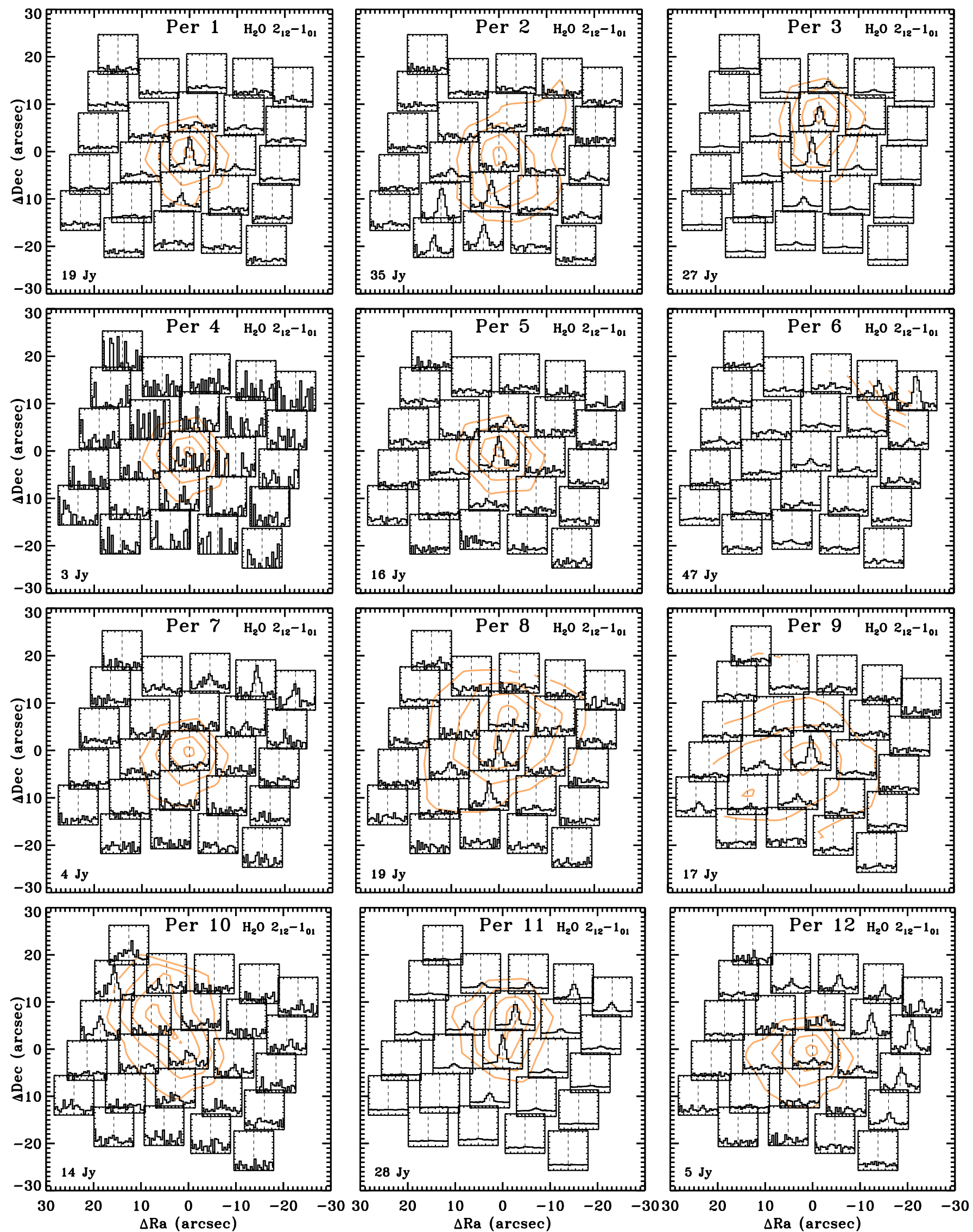

Fig. A.1. PACS spectral maps in the $\mathrm{H}_{2} \mathrm{O} 2_{12}-1_{01}$ line at $179.527 \mu \mathrm{m}$. Wavelengths in microns are translated to the velocity scale on the $X$-axis using laboratory wavelengths of the species and cover the range from -600 to $600 \mathrm{~km} \mathrm{~s}^{-1}$. The $Y$-axis shows fluxes normalized to the spaxel with the brightest line on the map in a range -0.2 to 1.2 . The orange contours show continuum emission at $30 \%, 50 \%, 70 \%$, and $90 \%$ of the peak value written in the bottom left corner of each map. 

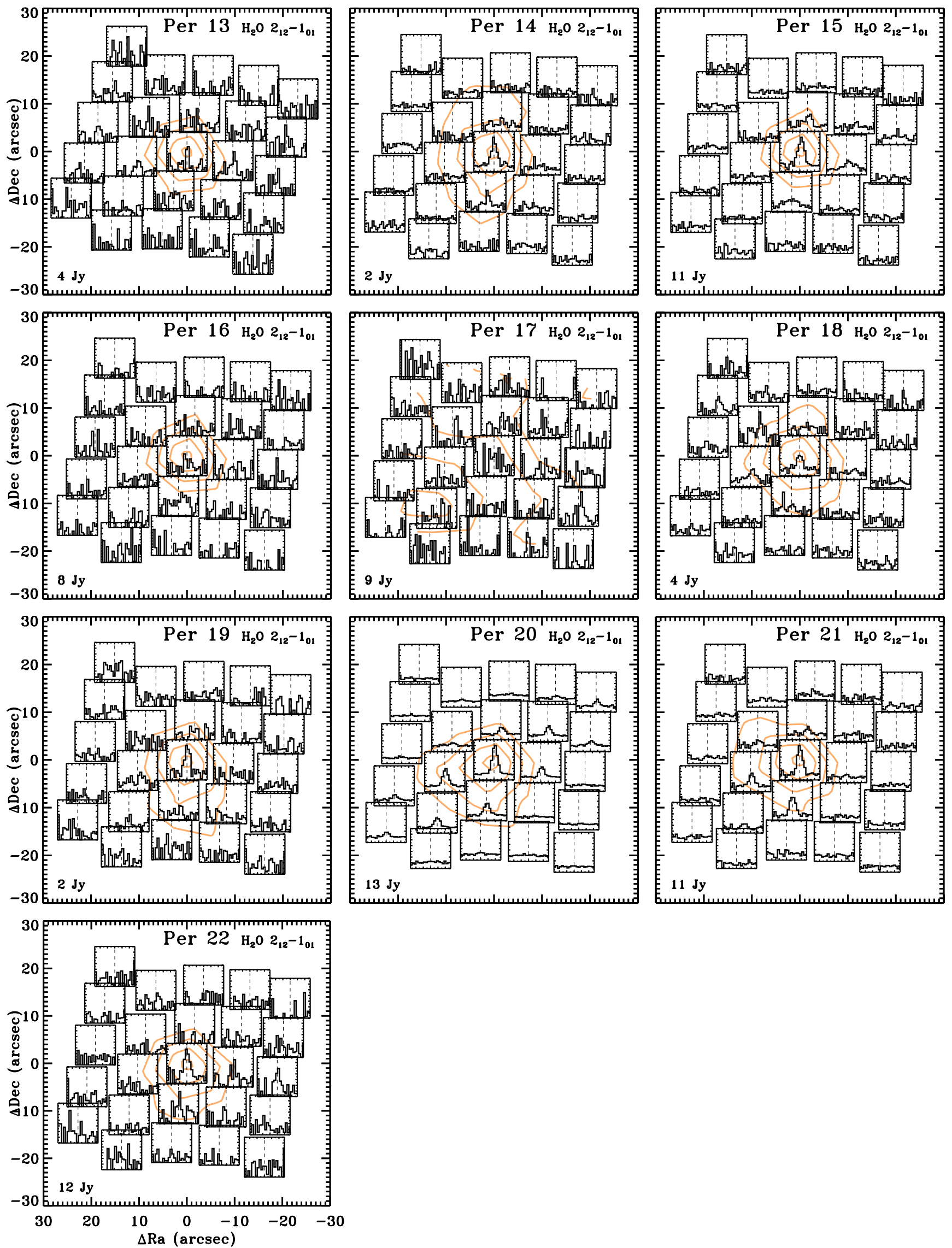

Fig. A.2. Same as Fig. A.1 but for the remaining sources. 

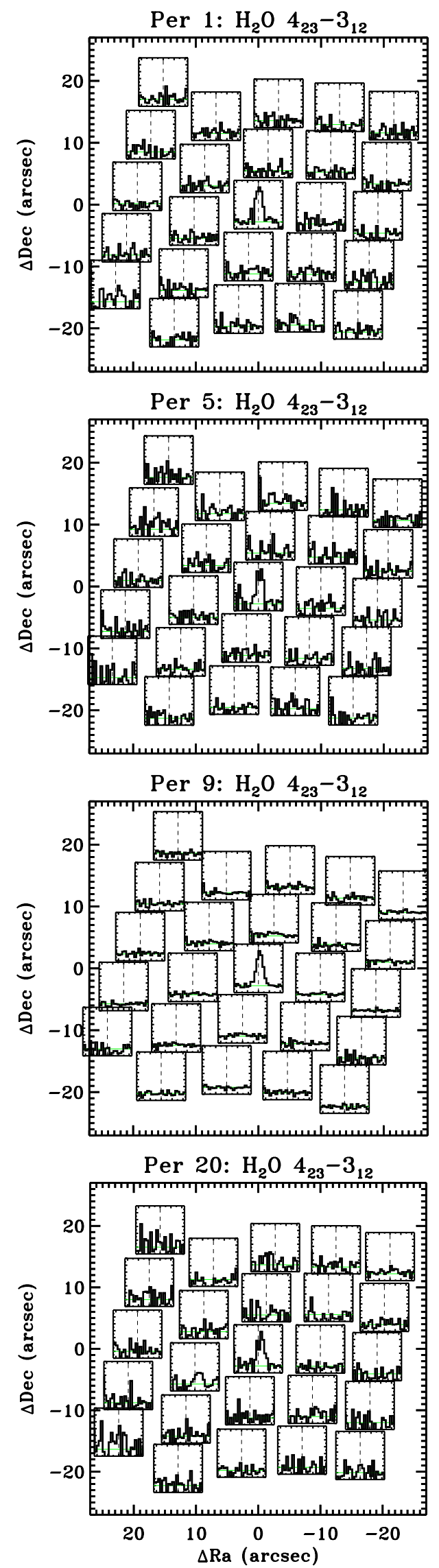

Per 1: $0 \mathrm{H} 84 \mu \mathrm{m}$

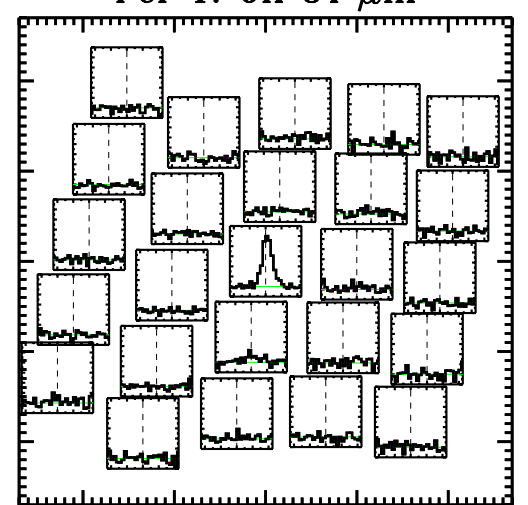

Per 5: $0 \mathrm{H} 84 \mu \mathrm{m}$

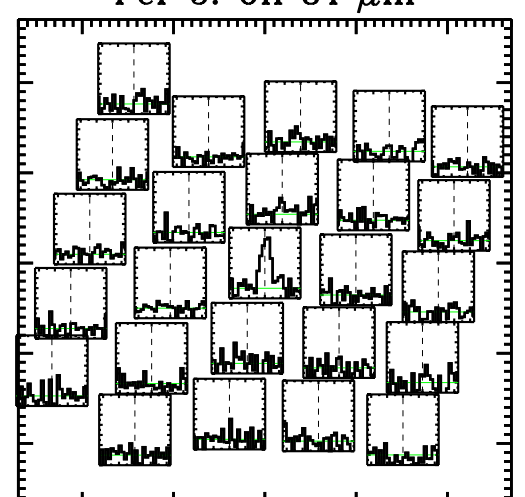

Per 9: $0 \mathrm{H} 84 \mu \mathrm{m}$

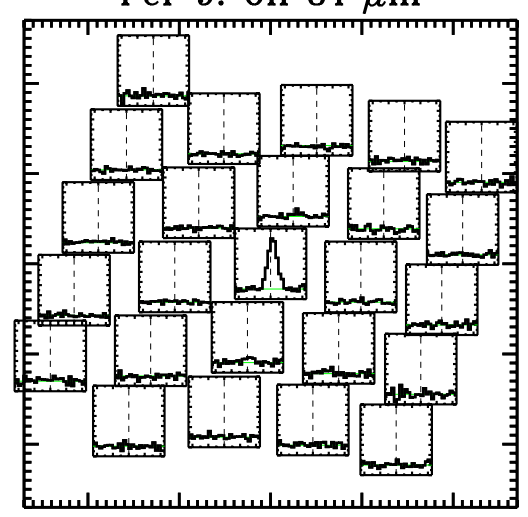

Per 20: $0 \mathrm{H} 84 \mu \mathrm{m}$

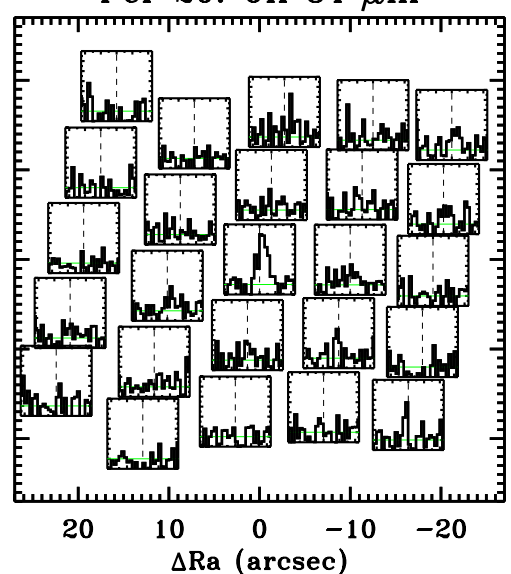

Per 1: CO 29-28

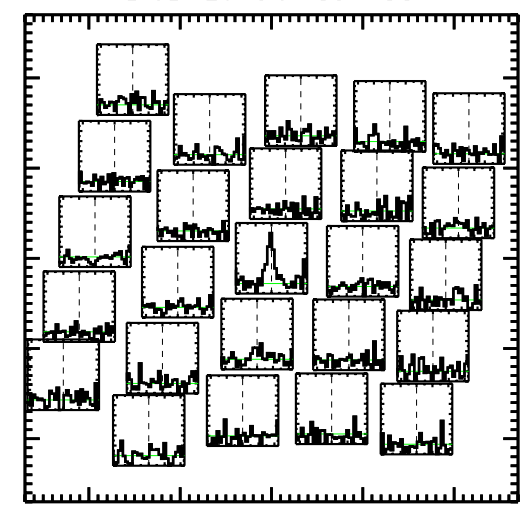

Per 5: C0 29-28

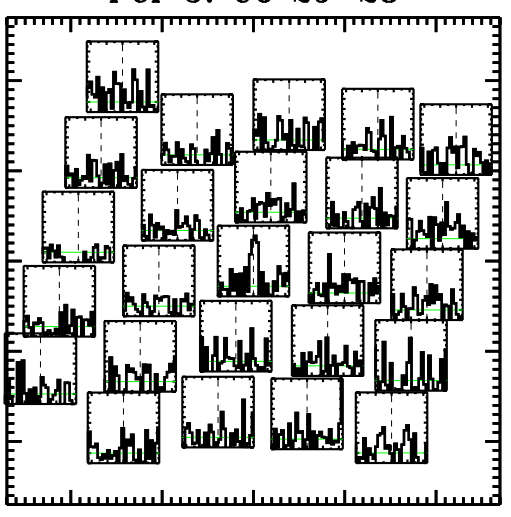

Per 9: CO 29-28

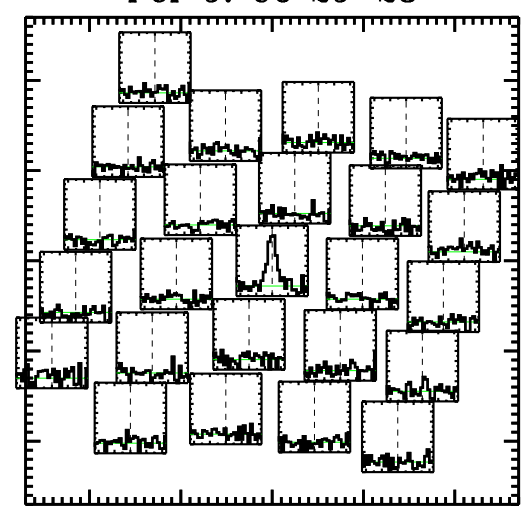

Per 20: CO 29-28

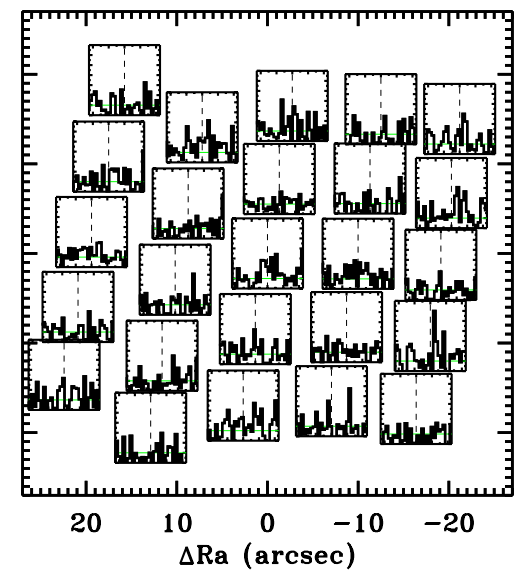

Fig. A.3. PACS spectral maps in the $\mathrm{H}_{2} \mathrm{O} 4_{23}-3_{12}$ line at $78.74 \mu \mathrm{m}$, the $\mathrm{OH}$ line at $84.6 \mu \mathrm{m}$, and the $\mathrm{CO} 29-28$ line at $90.16 \mu \mathrm{m}$ for a few sources with bright line emission and centrally peaked continuum. The $x$-axis range covers -450 to $450 \mathrm{~km} \mathrm{~s}^{-1}$ and the $y$-axis range covers -0.2 to 1.2 , with line emission normalized to the emission in the brightest spaxel. 


\section{Appendix B: Correlations with source parameters}

Figure B.1 shows selected $\mathrm{H}_{2} \mathrm{O}$-to-CO line ratios as a function of source physical parameters (bolometric luminosity, temperature, and envelope mass). Lack of correlation is seen in all cases.

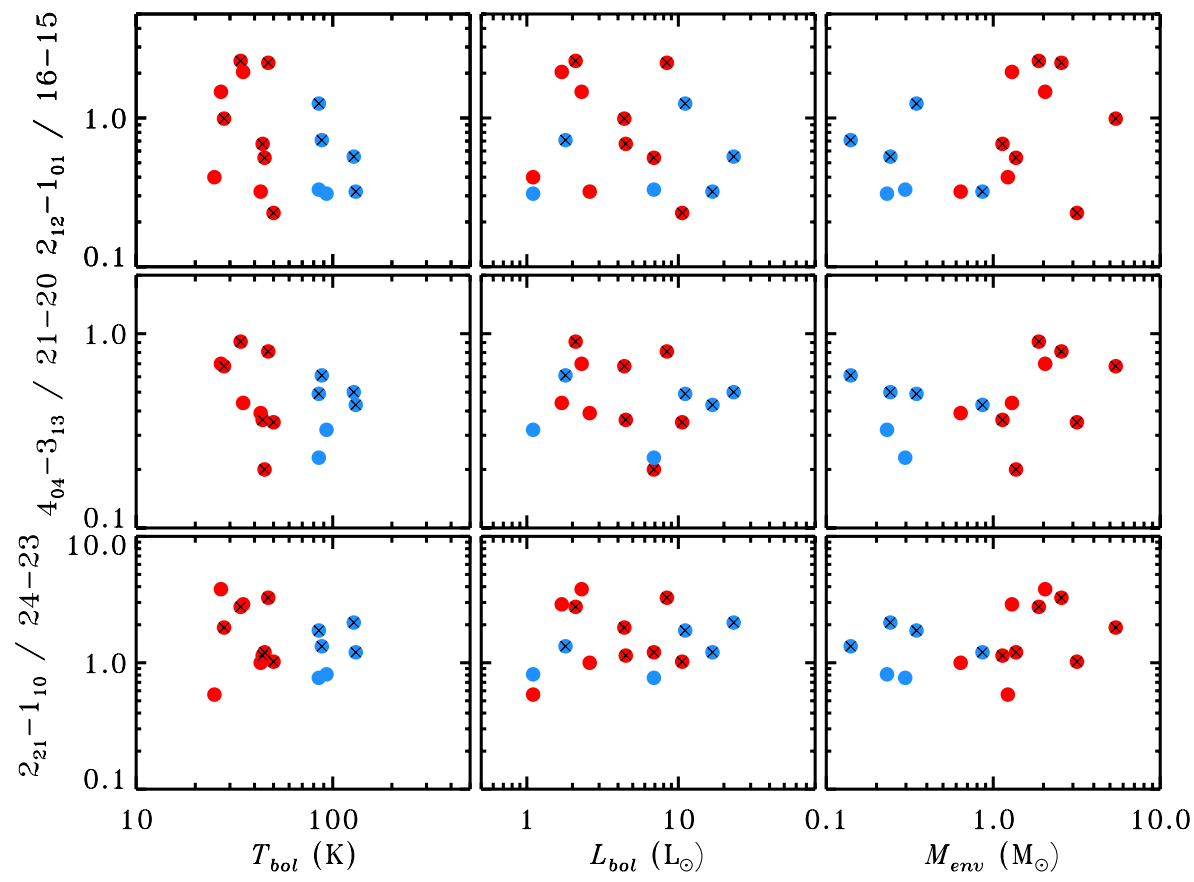

Fig. B.1. $\mathrm{H}_{2} \mathrm{O}$-to-CO line ratios and, from left to right, bolometric temperature, bolometric luminosity, and envelope mass. The ratios are calculated using lines at nearby wavelengths for which the same, limited to the central spaxel, extraction region is considered. Shown are line ratios of the $\mathrm{H}_{2} \mathrm{O} 2_{12}-1_{01}$ line at $179 \mu \mathrm{m}$ and $\mathrm{CO} 16-15$ at $163 \mu \mathrm{m}($ top $)$, the $\mathrm{H}_{2} \mathrm{O} 4_{04}-3_{13}$ and CO 21-20 lines at about $125 \mu \mathrm{m}$ (middle), and the $\mathrm{H}_{2} \mathrm{O} 2_{21}-1_{10}$ and CO 24-23 lines at about $108 \mu \mathrm{m}$ (bottom). Red and blue symbols correspond to sources with $T_{\text {bol }} \leq 70$ and $>70 \mathrm{~K}$, respectively. Black crosses are drawn for the sources with detections of CO 29-28 line. 